\title{
GATA-3: an unexpected regulator of cell lineage determination in skin
}

\author{
Charles K. Kaufman, ${ }^{1,5}$ Ping Zhou, ${ }^{2}$ H. Amalia Pasolli, ${ }^{1}$ Michael Rendl, ${ }^{1}$ Diana Bolotin, ${ }^{1}$ \\ Kim-Chew Lim, ${ }^{3}$ Xing Dai, ${ }^{4}$ Maria-Luisa Alegre, ${ }^{2}$ and Elaine Fuchs ${ }^{1,6}$ \\ ${ }^{1}$ Howard Hughes Medical Institute and Laboratory of Mammalian Cell Biology and Development, The Rockefeller \\ University, New York, New York 10021, USA; ${ }^{2}$ Department of Medicine, The University of Chicago, Chicago, Illinois \\ 60637, USA; ${ }^{3}$ University of Michigan Medical School, Ann Arbor, Michigan 48109, USA; ${ }^{4}$ Department of Biological \\ Chemistry, University of California, Irvine, Irvine, California 92697, USA
}

Multipotent skin stem cells give rise to epidermis and its appendages, including the hair follicle. The Lef-1/Tcf family of Wnt-regulated transcription factors plays a major role in specification of the hair shaft, but little is known about how the equally important hair channel, the inner root sheath (IRS), develops in concert to shape and guide the hair. In a microarray screen to search for transcriptional regulators of hair follicle morphogenesis, we identified GATA-3, a key regulator of T-cell lineage determination. Surprisingly, this transcription factor is essential for stem cell lineage determination in skin, where it is expressed at the onset of epidermal stratification and IRS specification in follicles. GATA-3-null/lacZ knock-in embryos can survive up to embryonic day 18.5 (E18.5), when they fail to form the IRS. Skin grafting unveiled additional defects in GATA-3-null hairs and follicles. IRS progenitors failed to differentiate, whereas cortical progenitors differentiated, but produced an aberrant hair structure. Curiously, some GATA-3-null progenitor cells expressed mixed IRS and hair shaft markers. Taken together, these findings place GATA-3 with Lef-1/Wnts at the crossroads of the IRS versus hair shaft cell fate decision in hair follicle morphogenesis. This newfound function for GATA-3 in skin development strengthens the parallels between the differentiation programs governing hair follicle and lymphocyte differentiation.

[Keywords: GATA-3; hair follicle development; Wnts; TGF- $\beta$ s; matrix; IRS]

Received May 20, 2003; revised version accepted June 25, 2003.

Hair follicles are derived from a single layer of multipotent embryonic ectoderm that produces the epidermis and its appendages. At the center of the mature follicle is the hair shaft, composed of a core, or medulla, surrounded by a concentric ring of cortical cells, and cloaked by a single layer of hair shaft cuticle (the hair surface). Beneath the skin surface, the hair shaft is surrounded by a channel, or inner root sheath (IRS), composed of three morphologically and biochemically discrete cell layers: the IRS cuticle, the Huxley's layer, and the Henle's layer. The IRS cuticle cells mesh like gears with the hair shaft cuticle cells, but near the skin surface, the IRS degenerates to liberate the hair (Dry 1926; Hardy 1992).

Both the hair shaft and the IRS arise through upward terminal differentiation of a population of transiently dividing progenitor cells, which are derived from the matrix, located at the base of the follicle. Matrix cells maintain their undifferentiated, proliferative state through in-

\footnotetext{
${ }^{5}$ Present address: The University of Chicago, 5841 S. Maryland Avenue, Chicago, IL 60637, USA.

${ }^{6}$ Corresponding author.

E-MAIL fuchslb@rockefeller.edu; FAX (212) 327-7954.

Article published online ahead of print. Article and publication date are at http://www.genesdev.org/cgi/doi/10.1101/gad.1115203.
}

teraction with a cluster of specialized mesenchymal cells, the dermal papilla (DP), at the core of the hair bulb (Oliver and Jahoda 1988; Cotsarelis et al. 1990). The entire follicle structure is enveloped by an outer root sheath (ORS), contiguous with and biochemically similar to the basal layer of the epidermis. Within the ORS in a region called the bulge, a reservoir of epidermal stem cells resides that is able to repopulate both epidermal and hair follicle lineages (Cotsarelis et al. 1990; Taylor et al. 2000; Oshima et al. 2001).

The accessibility, dispensability, and self-renewal features, coupled with its highly organized and fascinating architecture, have made the hair follicle a paradigm for exploring the molecular mechanisms that underlie stem cell lineage determination and complex differentiation programs that involve mesenchymal-epithelial interactions. A myriad of signal transduction pathways, including TGF- $\beta$ s, Bone morphogenetic proteins (Bmps), Sonic hedgehog (Shh), and Wnts, provide external cues that orchestrate cell fate decisions during hair follicle morphogenesis (for review, see Fuchs et al. 2001; Panteleyev et al. 2001; Millar 2002; Nieman and Watt 2002).

The hair shaft lineage seems especially sensitive to Wnt signaling, as substantiated by restricted expression of a Wnt reporter gene in the precortex of mature fol- 
licles (DasGupta and Fuchs 1999) and by the presence of matrix-hair shaft lineage tumors (pilomatricomas) in mice and humans displaying excessive stabilized $\beta$-catenin in skin epithelium (Gat et al. 1998; Chan et al. 1999; for review, see Millar 2002). Although much is known about hair shaft lineage determination, little is known about the parallel specification of the IRS, and yet this lineage is critical for the shaping and guidance of the hair to the skin surface. Given the overall importance of this channel to the hair, it seems highly unlikely that matrix cells would differentiate into IRS progenitors simply as a default pathway for those cells that do not become specified by Lef- 1 and $\beta$-catenin. Remarkably, however, of the myriad of transcription factors known to be expressed in the skin, only one, the CCAAT displacement protein (CDP), has been functionally linked to the IRS (Ellis et al. 2001). Even CDP's expression is not restricted to the IRS, as it is also found in the companion cell layer sandwiched between the ORS and IRS, and it is also in the outermost layer of the lower ORS. This broader zone of CDP expression may account for its severe effects when absent. CDP-deficient follicles not only exhibit a somewhat diminished IRS, but also an expanded ORS, misexpressed Sonic hedgehog and IRS genes, and cyst formation (Ellis et al. 2001).

In a search for novel transcription factors that might be implicated in cell lineage determination in the hair follicle, we compared the gene expression profiles of murine dorsal skin at three critical times during embryogenesis [embryonic days 13, 15, and 18.5 (E13, E15, E18.5); to be described in detail elsewhere]. The transcriptional regulator GATA-3 surfaced as a factor induced at E15 at the early stages of hair follicle placode formation and had sustained expression as the IRS began to develop at E18.

GATA-3 is a member of the GATA family of zinc finger transcription factors, which play key roles in controlling cell fate decisions, in particular, in different hematopoietic lineages (for review, see Kuo and Leiden 1999; Cantor and Orkin 2002). Early in lymphoid development, GATA-3 is essential for the T lymphoid cell lineage, whereas later, it is critical for differentiation of naive CD4+ T cells into Th2 as opposed to Th1 effector cells (Ting et al. 1996; Zhang et al. 1997; Zheng and Flavell 1997; Hendriks et al. 1999). The appearance of GATA-3 in our screen was particularly intriguing, because Lef-1, which is involved in hair shaft lineage determination, was named lymphoid enhancer factor 1 (Lef-1) on the basis of its initially described role in T-cell development, in which it regulates T-cell receptor genes (Carlsson et al. 1993; Giese and Groschedl 1993; van Genderen et al. 1994). This said, although ablation of GATA-3 results in severe defects in thymic and T-cell development, embryonic lethality arises from noradrenaline deficiency and cardiac defects, with additional abnormalities in cephalic neural crest and renal development (Pandolfi et al. 1995; Lim et al. 2000). Skin has never been examined in GATA-3-null embryos, which die by E11.5 without pharmacological rescue, prior to hair follicle morphogenesis.
In this report, we use a combination of genetics, cell biology, and biochemistry to assess whether GATA-3 might orchestrate lineage determination in the skin as it does in the immune system. We show that GATA-3 is expressed in a highly restricted fashion, in the epidermis and in the IRS of the hair follicle. By analyzing both pharmacologically rescued GATA-3-null embryos, which survive up to E18.5, and also grafted postnatal GATA-3null skin (Lim et al. 2000), we identify remarkable aberrations in hair follicle morphogenesis that include not only structural defects in the IRS and the hair shaft, but also molecular defects in cell lineage determination. We show here that loss of GATA-3, which is normally expressed in only two layers of the hair follicle IRS, results in follicles that produce an excess of IRS progenitor cells that fail to express IRS genes and fail to differentiate. In addition, follicles display an expanded compartment of Lef-1-positive, hair shaft progenitor cells. Although Shh is faithfully expressed, other abnormalities in gene expression arise, including some mixing of IRS and hair shaft markers. Finally, we show that in IRS and its progenitors, GATA-3 is coexpressed with Friend of GATA (FOG1), whose nuclear localization is lost in the GATA-3-deficient state.

\section{Results}

\section{GATA-3 expression during skin development}

When GATA-3 surfaced as an induced transcription factor in our Affymetrix microarray analyses of E13-E18.5 back skin mRNAs, we realized that skin has been previously included in a general list of GATA-3-positive organs (Oosterwegel et al. 1992; Lakshmanan et al. 1999). Because skin has $>20$ different cell types, we first evaluated GATA-3's temporal and spatial expression pattern in the skin. As judged by whole mount in situ hybridizations, GATA-3 cRNA hybridization was detected in the early developing vibrissae follicles by E14.5 (Fig. 1A). In body skin, hybridization was first detected at E15.5 (data not shown), and by E17, was evident both in the developing epidermis and hair follicles (Fig. 1B). These expression patterns extended our microarray analyses and revealed an induction of GATA-3 mRNAs in what appeared to be terminally differentiating cells of epidermis and the cone of presumptive IRS precursor cells (PreIRS) within developing follicles. The IRS develops somewhat earlier than the hair shaft, and precortical cells are not yet present at this stage.

To further analyze the temporal and spatial transcriptional changes in GATA-3 expression, we used the previously engineered GATA-3nlslacz mouse line, in which a gene encoding a nuclear-targeted version of $\beta$-galactosidase had been knocked in to the GATA-3 locus (Hendriks et al. 1999; van Doorninck et al. 1999). In mice heterozygous for this allele, $\beta$-galactosidase expression was absent in the E13 single-layered keratin K5-expressing embryonic epidermis but became evident in the newly formed suprabasal layer of E15 epidermis (Fig. 1C$\left.\mathrm{D}^{\prime}\right)$. Curiously, although $\beta$-galactosidase and GATA- 
Kaufman et al.

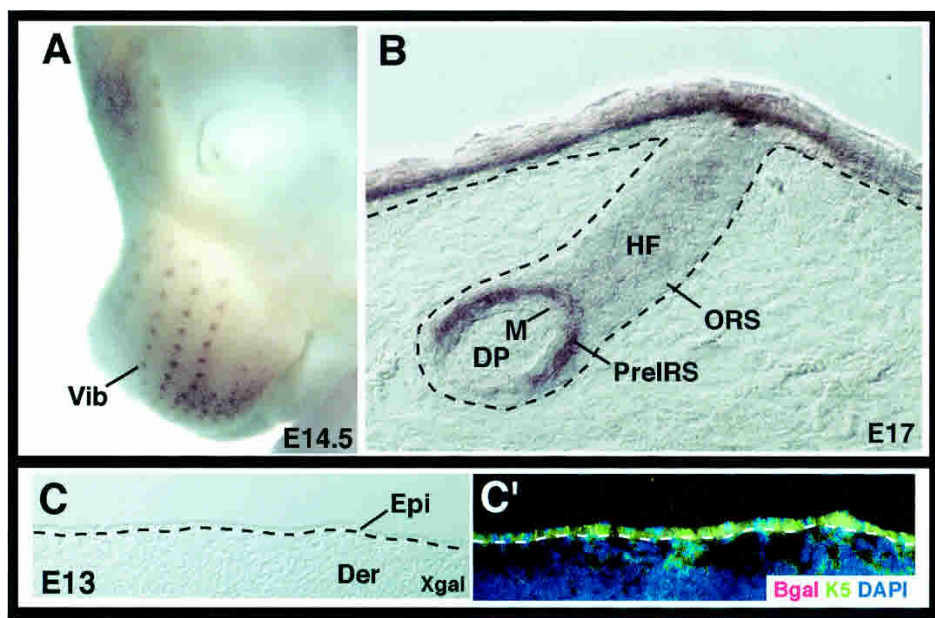

Figure 1. GATA-3 is expressed in embryonic skin epithelium. $(A, B)$ Whole mount in situ hybridization with a GATA-3 digoxygenin cRNA probe (brown). (A) Whole embryo hybridization, depicting GATA-3 mRNA in developing vibrissae (Vib) at E14.5. (B) Frozen section of skin from E17 whole mount in situ hybridization. Note strong hybridization in suprabasal epidermis and progenitor cells for the IRS (preIRS). (C-E) Frozen sections of GATA$3 n l s l a c Z /+$ embryos, at ages indicated in lower left of each frame. Sections were subjected to either X-gal staining $(C, D, E)$ or immunofluorescence $\left(C^{\prime}, D^{\prime}, E^{\prime}\right)$ with DAPI to detect nuclei, and with antibodies (Abs) against $\beta$-galactosidase (Bgal) to detect GATA-3 promoter activity and against K5 to detect the basal epidermal layer and follicle ORS. Abs are color-coded in the lower right of each frame. (Epi) Epidermis; (Der) dermis; (HF) hair follicle; (DP) dermal papilla; (ORS) outer root sheath; (M) matrix.

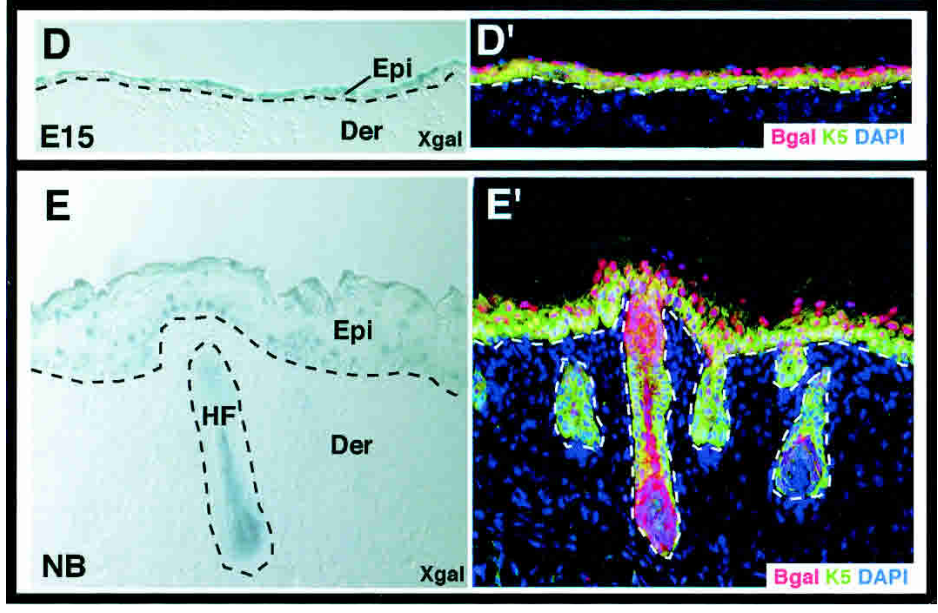

3nlslacZ expression were either absent or weak within the E15 embryonic basal layer (Fig. 1D, $\mathrm{D}^{\prime}$ ), it was restored in this layer of newborn epidermis and remained on thereafter (Fig. 1E, $E^{\prime}$ ). Overall, expression patterns of GATA-3 mRNAs and GATA-3 promoter-driven nlslacz activity were similar if not identical.

By postnatal day $16(\mathrm{P} 16)$, when back skin follicles are mature and nearing completion of the first growth (anagen) phase of the hair cycle (Hardy 1992), GATA-3 was clearly expressed in follicles as well as epidermis (Fig. $\left.2 \mathrm{~A}, \mathrm{~A}^{\prime}\right)$. Shortly after the synchronous initiation of the first postnatal hair cycle, X-gal staining was seen in a cone, distinctive of the organization of PreIRS, composed of IRS progenitor cells (Fig. 2B, $\mathrm{B}^{\prime}$ ).

To determine the precise location of GATA-3 expression within the hair follicle, we compared GATA-3 promoter and protein expression to the expression of wellestablished biochemical markers for follicles. As judged by both GATA-3-promoter-driven $\beta$-galactosidase expression and GATA-3 protein expression, GATA-3 expression was restricted to two cell layers of the follicle (green in Fig. 2C-E). These layers were located directly external to the hair shaft cuticle and cortex/precortex, marked by an AE13 monoclonal antibody specific for hair-specific keratins (Fig. 2C, red; Lynch et al. 1986). At the base of the follicle, the two $\beta$-galactosidase-positive layers were directly internal to a single layer of IRS cells labeling with the monoclonal antibody AE15 (Fig. 2D, red) specific for trichohyalin, a marker of IRS and postnatal, differentiating medulla $(\mathrm{Me})$ cells $\left(\mathrm{O}^{\prime}\right.$ Guin et al. 1992). Further up, the two $\beta$-galactosidase-positive cell layers colabeled with AE15 antibodies, which now detected all three IRS layers.

Furthermore, a single layer of unlabeled nuclei (Henle's layer) was present between the K6-positive companion cell layer and the two GATA-3-positive Huxley's and cuticle layers of IRS (Fig. 2E). Although some K6 genes are also expressed in IRS (Winter et al. 1998; Langbein et al. 2003), the K6 antibody used here did not recognize these isoforms. In addition, two cell layers, namely, the hair shaft cuticle $(\mathrm{Ch})$ and the cortex (Co), separated AE15- and K6-positive medulla from GATA-3-positive IRS cuticle (Fig. 2D,E). The diagram in Figure 2 summarizes the localization of markers relative to GATA-3 promoter and GATA-3 protein expression.

\section{Defects in GATA-3-null embryonic skin}

With a firm grasp of the skin expression patterns of GATA-3, we began an analysis of GATA-3-null epidermis in E18.5 embryos, the latest stage to which these animals survive. At this stage, back skin epidermis is 


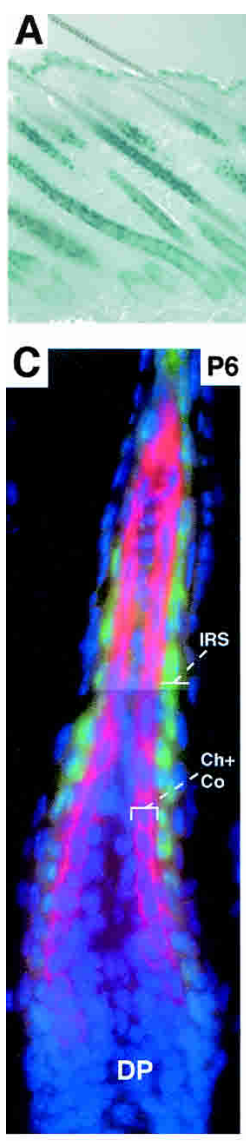

Bgal AE13 DAPI

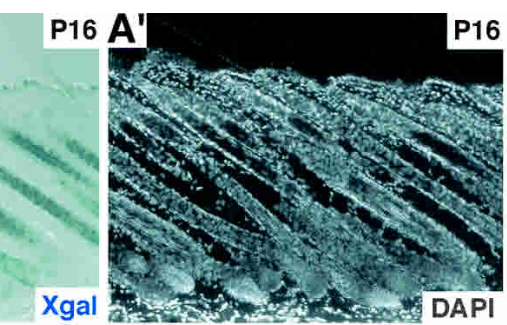

P16 B
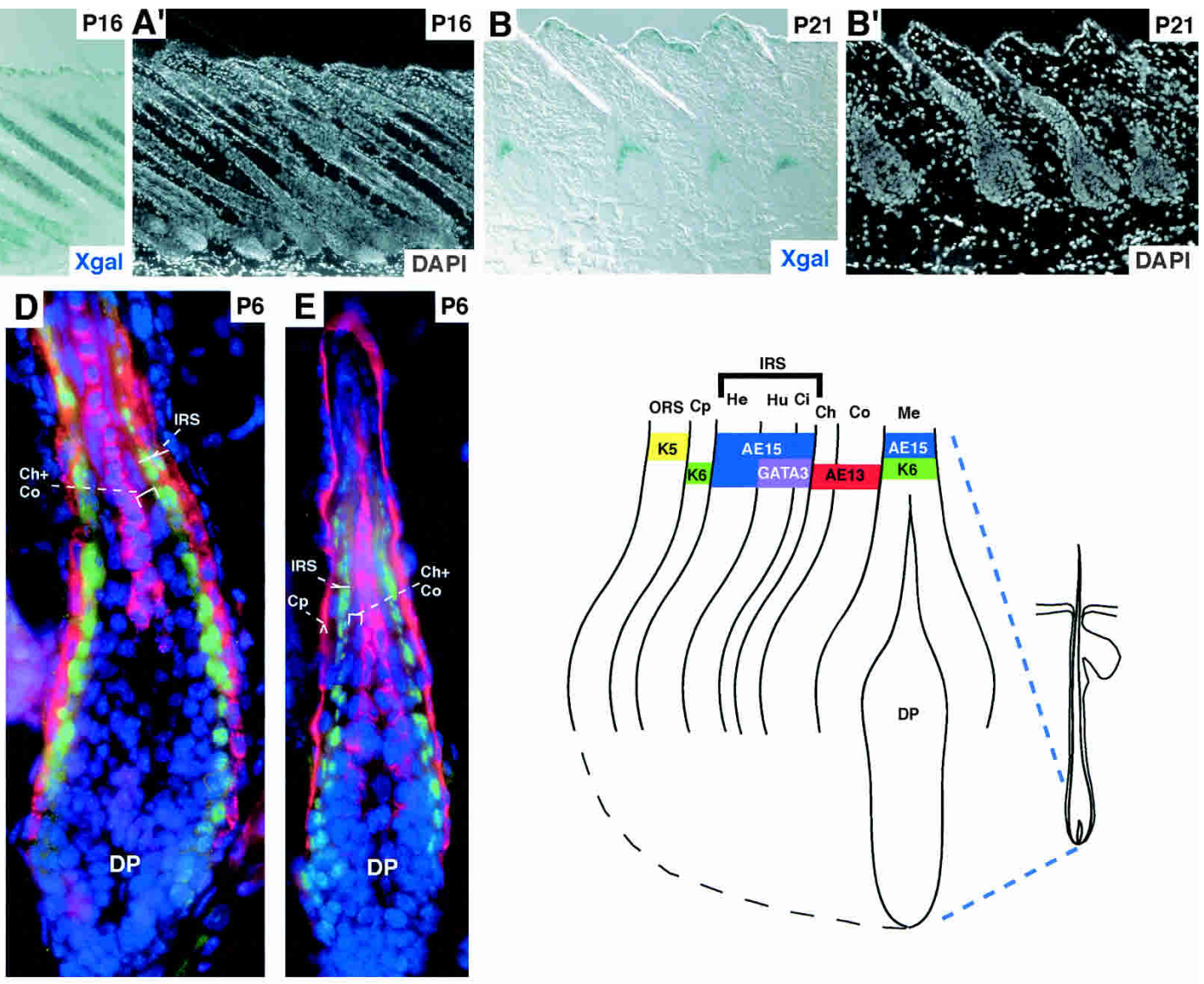

Bgal AE15 DAPI

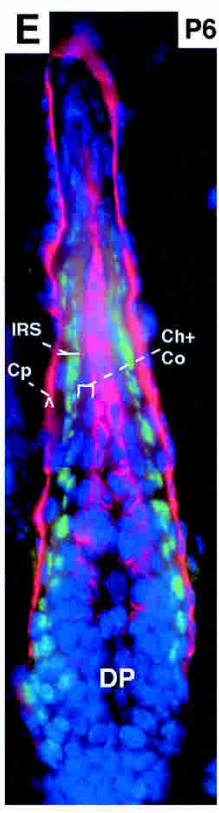

GATA-3 K6 DAPI

\section{西}

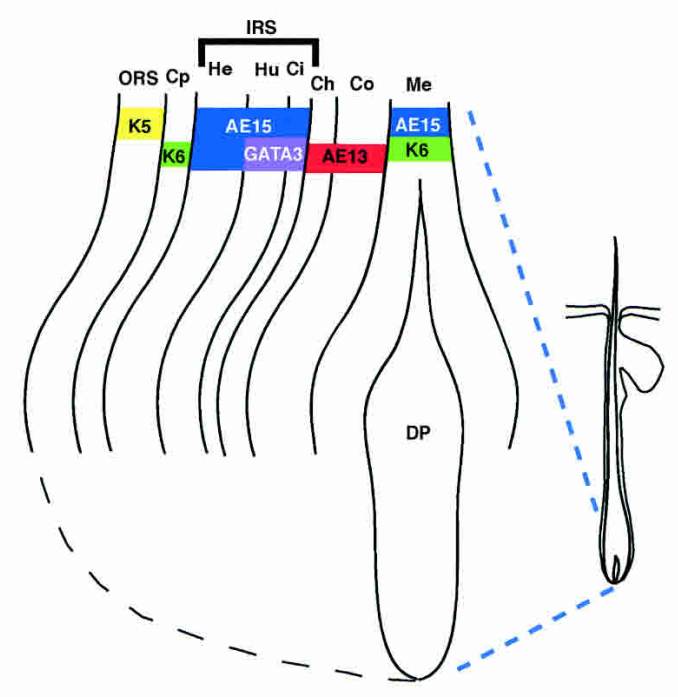

Figure 2. GATA-3 expression correlates with IRS differentiation. Dorsal skins were from GATA-3nlslacz/+ mice at the postnatal days indicated in the upper right of each frame. Skins were frozen, sectioned, and stained with DAPI and X-gal $(A, B)$, or subjected to indirect triple immunofluorescence (C-E) with DAPI and Abs against $\beta$-galactosidase (Bgal) to detect GATA-3 promoter activity; AE13 to detect cortical acidic hair keratins; AE15 to detect IRS and medulla trichohyalin; K6 to detect the companion layer (Cp) keratins and the medulla K6 keratins; or GATA-3. Antibodies are color-coded as indicated on each frame. The stages of the hair cycle are as follows: P16, late anagen, first cycle; P21, early anagen, second cycle; P6, early to mid-anagen, first cycle. $(A, B) 20 \times$ magnification, $10-\mu m$ sections. $(C-E)$ Composites of two microscopic fields at $63 \times$ magnification, $6-\mu \mathrm{m}$ sections. (ORS) outer root sheath; (Cp) companion cell layer; (IRS) inner root sheath; (He) Henle's layer; (Hu) Huxley's layer; (Ci) cuticle of IRS; (Ch) cuticle of hair shaft; (Co) cortex of hair shaft; (Me) medulla; (DP) dermal papilla. The diagram at right summarizes the immunofluorescence patterns observed within the bulb of the hair follicle. The K6 antibody we use here does not recognize the IRS-specific K6 keratins.

mature, and most waves of follicle morphogenesis have initiated. However, because the most mature follicles in the E18.5 embryo are vibrissae, we began by examining whisker pads.

Whiskers of control littermates were visible on E18.5 embryos, and hematoxylin and eosin (H\&E) staining of wild-type vibrissae sections revealed morphological signs reflective of mature follicles (Fig. 3A). In E18.5 wild-type vibrissae, the precortex was labeled with AE13 antibodies, indicative of hair keratin expression (Fig. $3 \mathrm{Ai}, \mathrm{Bi}$ ). Analogous to postnatal follicles, several cell layers separated AE13-positive hair shaft cells from the K6positive companion cell layer (separation marked by bracket in Fig. 3Ai,Bi). Serial sectioning revealed that, as expected, cells between the hair shaft and companion cell layer are IRS cell layers, which stained positively for the trichohyalin antibody AE15 (Fig. 3Aii,Bii).

In E18.5 GATA-3-null vibrissae, AE13 (hair keratin)- positive cells appeared to directly abut the K6-positive cells of the companion cell layer (Fig. 3Ci,Ciii,Di,Diii). By staining alternating serial sections with AE13 and AE15 antibodies, it was clear that AE15-positive cells, characteristic of IRS, were either absent or severely reduced in GATA-3-null E18.5 vibrissae (Fig. 3Cii,Dii). In addition, the AE13-positive hair shaft and associated precursor layers appear to be expanded in GATA-3-null follicles relative to wild-type follicles (cf. AE13 staining in Fig. 3Ai and in 3Ci,Ciii,Di,Diii). These abnormalities were seen in all vibrissae, irrespective of their location within the GATA-3-null whisker pad (two examples are shown).

Morphological aberrations in GATA-3-null vibrissae follicles included atypically bent shapes, strange nodular thickenings (asterisk in Fig. 3C), and irregularities in the thickness of the ORS and bulb (Fig. 3C,D). These perturbations were manifested at the skin surface by a delayed 


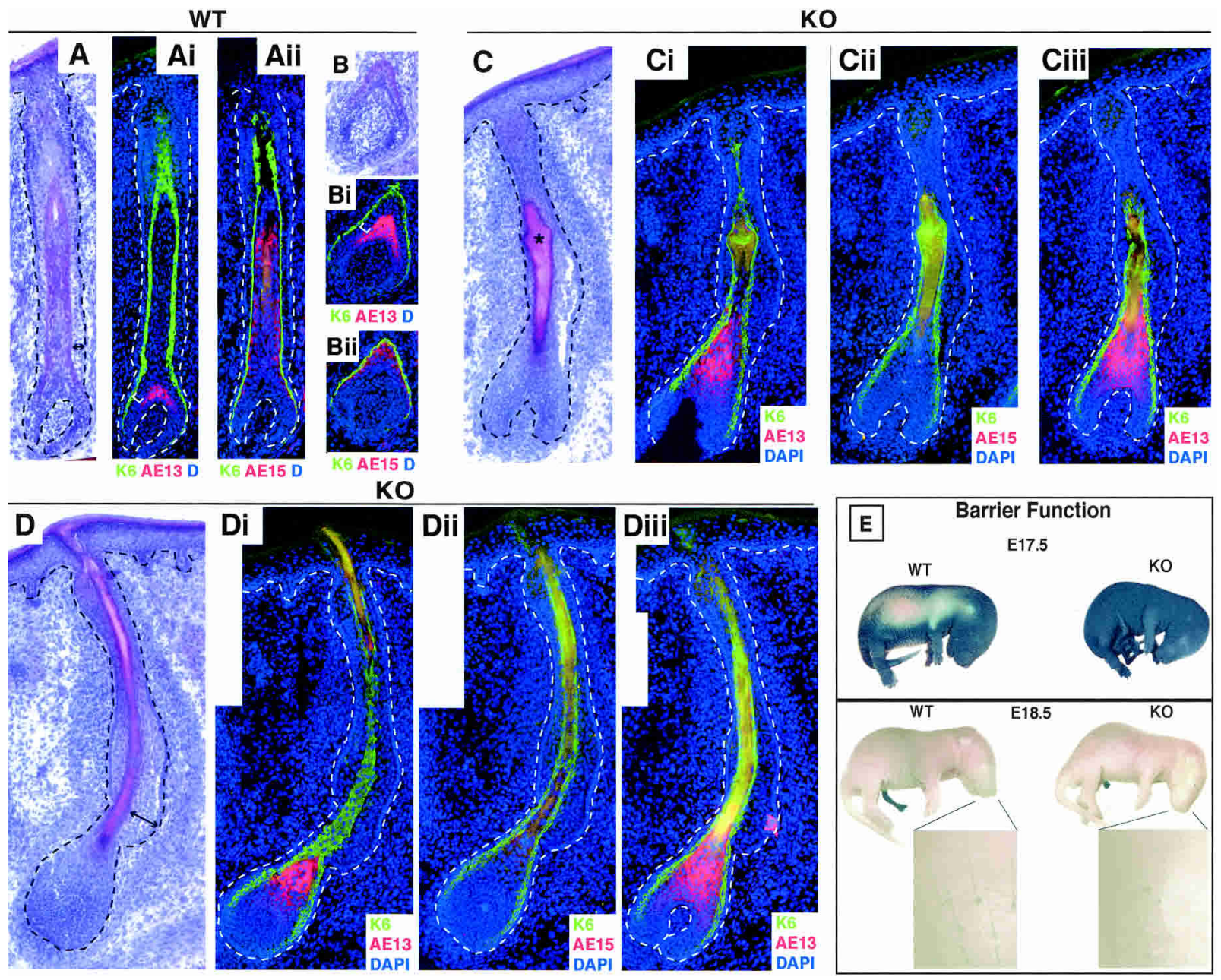

Figure 3. Whisker follicles develop by E18.5, but they are aberrant in GATA-3-null embryos. Whisker pads of E18.5 wild-type (WT) and GATA-3nlslacz(z/z) (GATA-3-null) embryos were frozen and sectioned (10 $\mu \mathrm{m})$. Serial sections (denoted by i, ii, iii) of two wild-type follicles $(A, B)$ and two KO follicles $(C, D)$ are shown. Sections were either subjected to H\&E staining $(A-D)$ or triple indirect immunofluorescence microscopy using the antibodies indicated in the lower right of each frame. Color coding reflects the secondary Abs used for detection. Brackets in Ai and Bi denote a gap between the hair cortex (red, AE13) and companion cell layer (green, K6). This gap represents the IRS (red, AE15; Aii and Bii), which is sandwiched between the companion layer and the cortex of wild-type follicles. In KO follicles, AE15 staining is largely absent, and K6 and AE13 staining are adjacent to one another. Additional abnormalities in embryonic KO follicles include nodular thickenings in the vibrissae shafts (marked with an asterisk; $C$ ), irregular bulges in the ORS (e.g., double-headed arrow; $D$ ), and a shortened, bent follicle of enlarged diameter at the bulb. Images are shown at 20x magnification. Panels are composites of two or three microscopic fields. $(E)$ Barrier function analyses. The ability of whole embryos to exclude blue dye was used to examine the epidermal barrier, normally acquired beginning at E17.5 and complete by E18.5. Note the complete penetration of blue dye in a representative GATA-3-null E17.5 embryo, in contrast to the comparably sized E17.5 wild-type embryo, which has the characteristic dorsal pattern of dye exclusion. By E18.5, both wild-type and null embryos have acquired barrier function based on this assay. Note, however, that whiskers have not protruded from the muzzle of the KO embryo, in contrast to the wild-type littermate.

eruption of GATA-3-null relative to wild-type vibrissae shafts (Fig. 3E, see insets).

Elsewhere within E18.5 skin, few differences were noted. As a measure of epidermal function, E18.5 knockout (KO) embryos were able to exclude blue dye, indicating that the epidermal barrier was intact (Fig. 3E). Analyses of E17.5 embryos revealed a slight delay in barrier function acquisition at this earlier age, as judged by the complete penetration of blue dye through GATA-3-null skin relative to only partial penetration through wild- type E17.5 skin (Fig. 3E). Overall, however, the morphology and biochemistry of GATA-3-null epidermis throughout E18.5 embryos was largely similar to wildtype epidermis (Fig. 4). Thus, basal (BL), spinous (SL), granular $(\mathrm{GL})$, and cornified $(\mathrm{CL})$ layers were all present and of comparable size and thickness. Wild-type and KO epidermis displayed normal staining for the basal layer keratin 5, the suprabasal layer keratin 1, and the latestage differentiation markers involucrin, loricrin, and filaggrin. 


\section{WT}

A
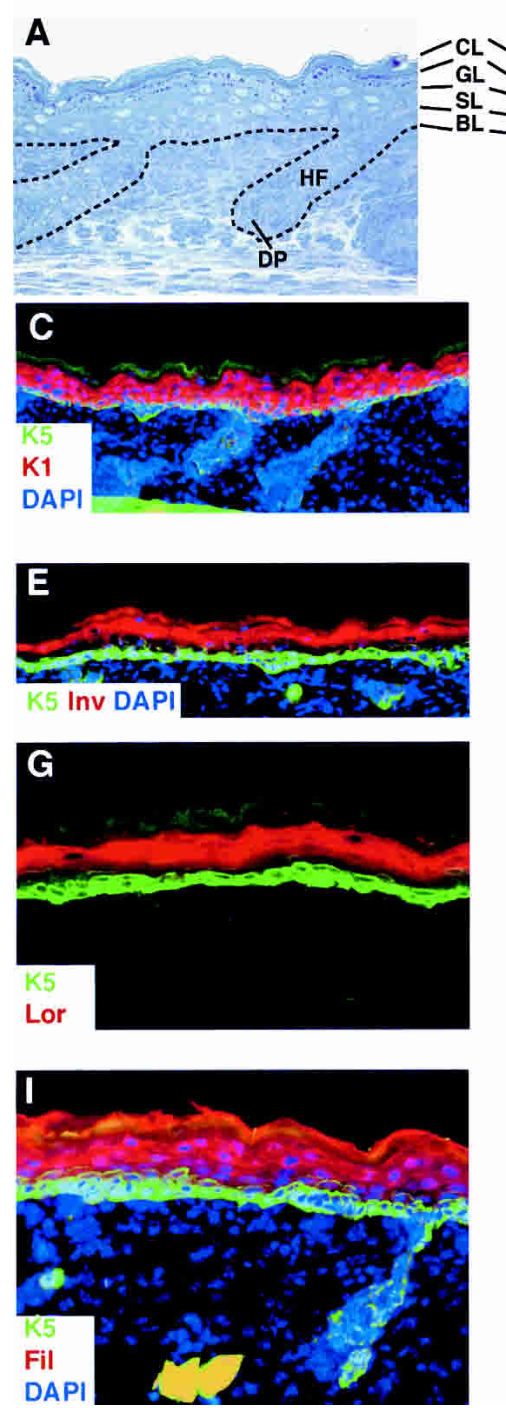

KO

B
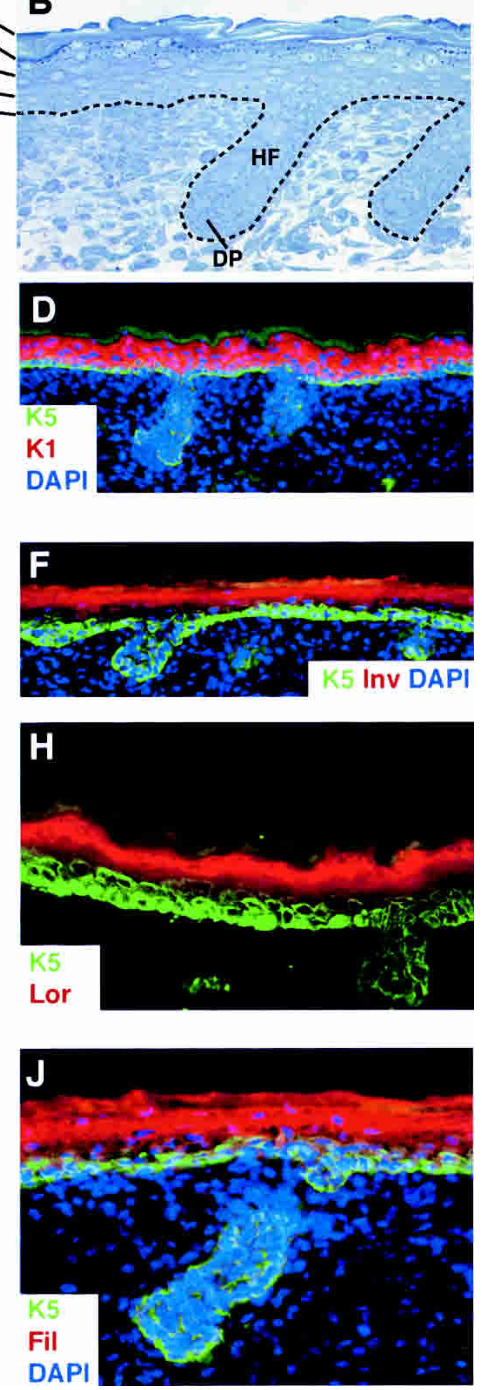

Figure 4. Classical morphological and biochemical features of epidermal differentiation are normal in GATA-3-null animals. Panels are from an E18.5 wildtype littermate (left set) and a GATA-3-null embryo (right set). $(A, B)$ Dorsal back skins were fixed in Epon and embedded, and semithin sections $(1 \mu \mathrm{m})$ were stained with toluidine blue. Note the presence of basal $(\mathrm{BL})$, spinous $(\mathrm{SL})$, granular $(\mathrm{GL})$, and cornified $(\mathrm{CL})$ layers, which are largely similar between wild-type and KO skin. Note also similarities in wild-type and KO pelage hair follicles (HF), which at this stage have not begun to differentiate into shaft, IRS, and ORS. (C-I) Frozen sections of E18.5 dorsal back skins were subjected to triple indirect immunofluorescence using DAPI to label the nuclei and the following antibodies: K5 to label the epidermal basal layer; K1 and involucrin (Inv; in mouse skin, Inv is more suprabasal than in human) to label the spinous layers; filaggrin (Fil) and loricrin (Lor) to mark the granular layers (Fil detects pre- and processed filaggrin). Similar normal biochemical patterns of staining were observed for mature epidermis, obtained from grafted $\mathrm{KO}$ and wild-type skins.
Morphological and biochemical signs of IRS and hair shaft differentiation are largely absent in back skin prior to birth (e.g., see Fig. 4A,B). The lack of overt abnormalities in the back skin of even the oldest living GATA-3 KO embryos explains why skin defects had not been noted previously in these animals (Lim et al. 2000).

\section{Skin grafting reveals gross defects in postnatal development of GATA-3-null hairs}

A priori, the selective abnormalities in E18.5 GATA-3null vibrissae could be attributed either to a special role for GATA-3 in vibrissae or to their early development. In addition, given the well-established role of GATA-3 in hematopoiesis and CNS/PNS development, it was possible that the hair follicle defects were attributable to indirect rather than autonomous effects. To distinguish between these possibilities, we grafted full thickness E17.5 skin from GATA-3-null and wild-type littermate embryos onto backs of recipient $n u / n u$ mice. Mice with the $n u / n u$ mutation are immunocompromised as they lack $\mathrm{T}$ cells and cannot reject the graft. They also lack nearly all external hair shaft formation, but are wild type for GATA-3 (Flanagan 1966; Nehls et al. 1994; Segre et al. 1995).

When bandages were removed from recipient mice at 7 $\mathrm{d}$ after engraftment, hair shafts were already evident on wild-type grafts but not on GATA-3-null grafts (Fig. $5 \mathrm{~A}, \mathrm{~B}$; examples shown are day 9 post engraftment). Grafts were monitored daily, and in all 6 of 6 grafts, KO skin consistently failed to develop the thick hair coat displayed by wild-type grafts (Fig. 5C-F). Instead, KO grafts developed short "stubble" that failed to progress into a normal hair coat (Fig. 5D,F).

In wild-type grafts, all three major hair types, including awl, guard, and zig-zag hairs, were readily identified upon microscopic examination of plucked hairs (Fig. 5G). In contrast, hairs from GATA-3-null grafts were 


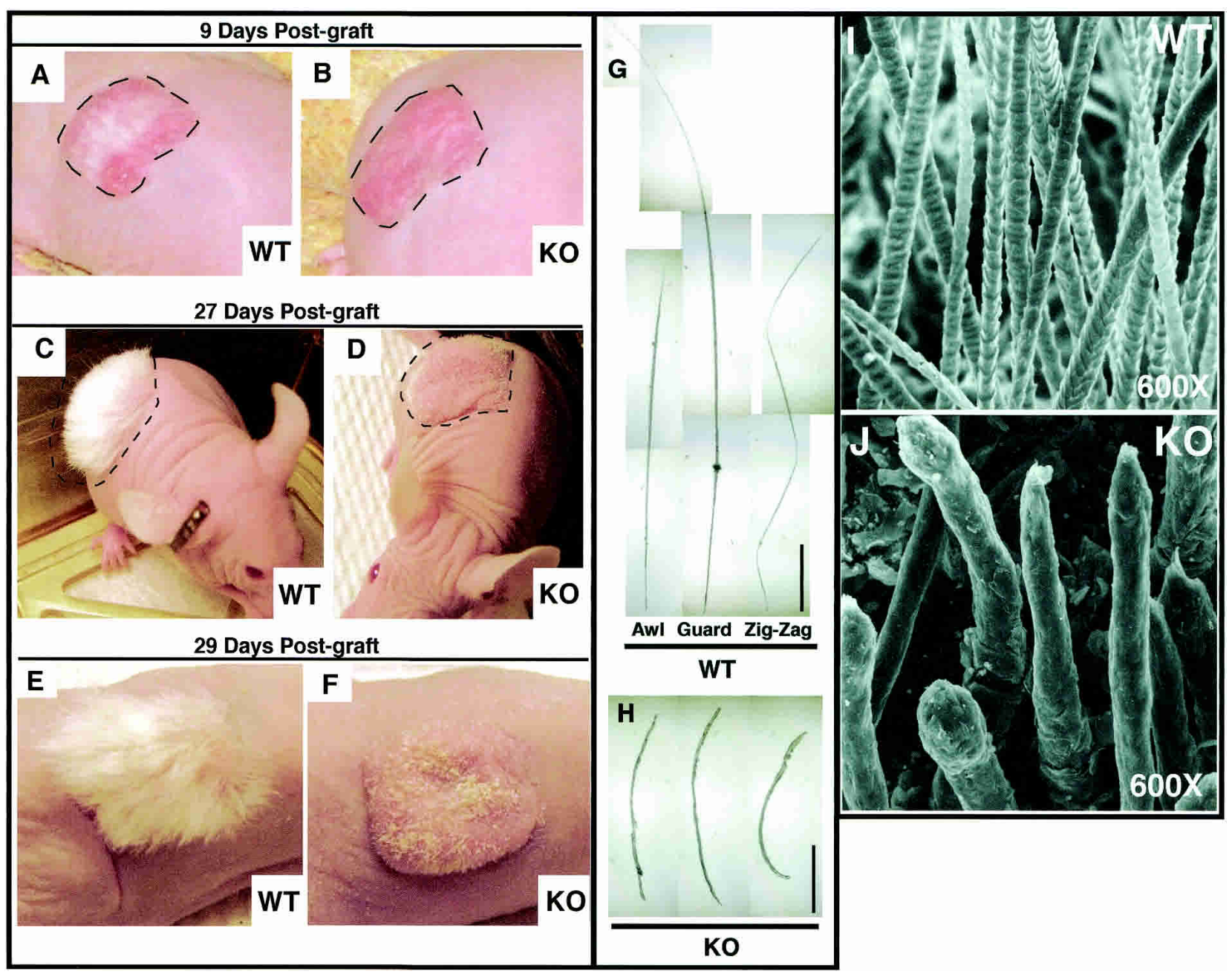

Figure 5. GATA-3-null skin grafts develop strikingly abnormal hairs. Full thickness skins from six wild-type and six GATA-3-null (KO) E17.5 CD-1 albino embryos were grafted onto nu/nu mice. At $9 \mathrm{~d}$ after engraftment, hairs (white) appeared in all wild-type grafts $(A)$ but were absent in all GATA-3-null grafts $(B)$. By 27-29 d, a thick pelage hair coat had developed on all wild-type grafts $(C$ and $E$ show representative examples of grafts from skins of two different wild-type animals), but the KO grafts produced only short, stubby hairs $(D$ and $F$ show representative examples of grafts from skins of two different KO animals). The three major hair types (awl, guard, and zig-zag) were evident among hairs plucked from wild-type grafts (examples shown in $G$ ) at 12 wk postengraftment. In contrast, hairs from equivalent GATA-3-null grafts (representative examples shown in $H$ ) did not fit these classifications and were shorter and thicker than wild-type shafts. $(I, J)$ Scanning electron microscopy (SEM) analysis. SEM $(600 \times)$ of the surface of wild-type and KO grafted skin at 12 wk postengraftment. The outer surface of each hair shaft is the cuticle layer, which is highly ordered in the wild-type but not KO mice. Note additional irregularities in the shafts, reflecting in detail the differences noted at lower magnification.

shorter and wider and did not fall into any standard classification (Fig. 5H). As judged by scanning electron microscopy, the fine structure of these hairs was highly irregular. Wild-type follicles displayed a "shingle-like" pattern, characteristic of flat cuticle cells that constitute the outer layer of normal hair shafts (Fig. 5I). Although flat cuticle cells were present in KO shafts, their organization was highly abnormal (Fig. 5J). Additionally, KO hair shafts were short, and their diameter was large and irregular compared with their wild-type counterparts. The nodules, bulges, and bends in GATA-3-null hairs paralleled the follicle defects seen below the skin surface in embryonic GATA-3-null vibrissae follicles (see Fig. 3).
Expansion of hair shaft and hair shaft precursor cells in GATA-3-null follicles

The skin grafts enabled us to examine changes in expression patterns of hair follicle proteins in mature pelage follicles, replete with hair shafts. Immunofluorescence analyses revealed severe abnormalities in the expression patterns of hair follicle proteins within GATA-3-null skin grafts (Fig. 6). As judged by AE13 staining (red) to detect the cortical keratins of hair shafts, GATA-3-null follicles exhibited a markedly expanded precortex and cortex as compared with wild-type grafts (Fig. 6A-D). These differences were further documented by a large increase in Lef-1-positive precortical cell nuclei in KO versus wild-type follicles (Fig. 6E,F). In addition, there 

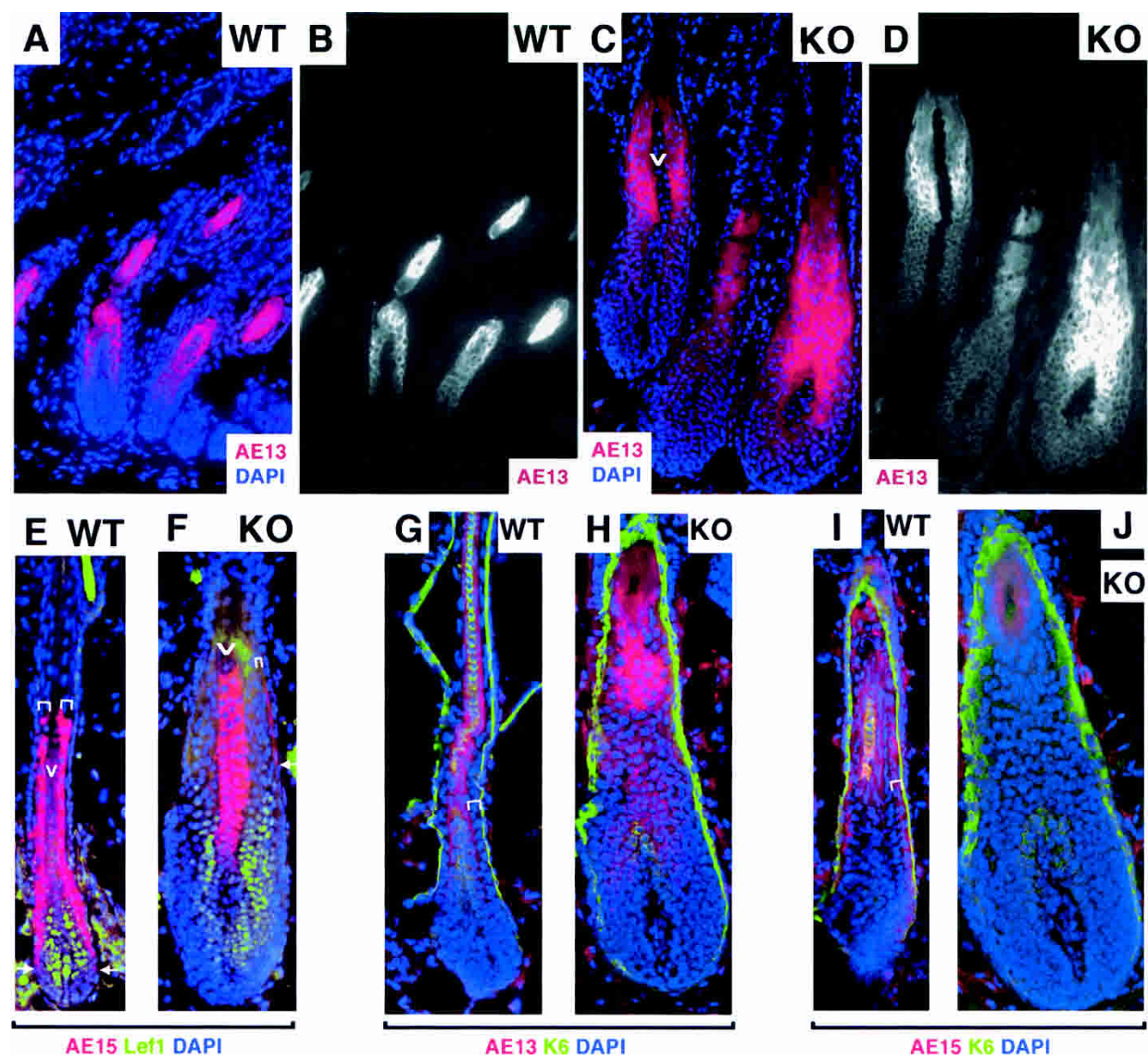

Figure 6. Mature GATA-3-null hair follicles exhibit biochemical signs of an expanded cortex and medulla and a severely reduced inner root sheath. $(A-J)$ Matched P35 skin grafts from E17.5 GATA-3-null (KO) and wild-type littermates were frozen and sectioned $(10 \mu \mathrm{m})$ and subjected to triple indirect immunofluorescence microscopy. Most follicles were in the anagen phase of the hair cycle. The upper right denotes genotype of skin. The antibodies used are noted on each frame and are color-coded according to the secondary $\mathrm{Ab}$ used for detection. DAPI was used to mark the nuclei, AE13 for cortical keratins, AE15 for IRS and medulla trichoyalin, anti-K6 for the companion layer and medulla (this antibody does not detect the IRS-K6 keratins), and Lef- 1 to mark the precortical cells. $\vee$ denotes the wide stripe of AE13-negative, AE15-positive cells, corresponding to the medulla at the hair shaft center. Note the expanded Lef-1-positive precortex and AE13-positive cortex in mutant follicles. Brackets denote the AE15-positive IRS, largely absent in the KO follicles. Arrows indicate the lowest extent of AE15-positive IRS staining.

was an expansion of differentiated medulla cells expressing trichohyalin (denoted by $\vee$ in Fig. $6 \mathrm{~F}$; cf. WT in Fig. $6 \mathrm{E}$ ), which was flanked by the AE13-positive cortex (cf. $\vee$ in Fig. 6C and 6F). Ultrastructural analyses later confirmed the identity of these cells as medulla rather than a misplaced core of IRS cells.

Paucity of differentiated IRS cells but expansion of IRS precursor cells in GATA-3-null skin

The increases in cortical and medulla cells were paralleled by a paucity of differentiated IRS cells labeled with AE15 antibodies against trichohyalin. Thus, in contrast to the two robust stripes of wild-type IRS stained with AE15 antibodies (denoted by brackets in Fig. 6E), only wisps of AE15-positive KO IRS cells were detected (bracket in Fig. 6F). Additionally, these remnants of what appeared biochemically to be IRS were always found well above the bulb of the follicle and above the initia- tion of medulla differentiation (see arrow in Fig. 6F). In marked contrast, IRS differentiation in wild-type grafts always initiated in a horizontal plane near the base of the bulb, comparable to that of the Lef-1-positive cortical precursors and well below the differentiating medulla (arrows in Fig. 6E).

Despite the paucity of trichohyalin-positive IRS cells of GATA-3-null grafted skin, the K6-positive companion layer appeared to be largely normal in size and location (Fig. 6G-J). However, whereas the companion layer in wild-type follicles flanked the AE13-negative/AE15positive IRS cells (marked with brackets in Fig. 6G,I), it flanked the AE13-positive/AE15-negative cortical cells in $\mathrm{KO}$ follicles (Fig. $6 \mathrm{H}, \mathrm{J}$ ). Overall, these results provide additional evidence that differentiating IRS cells are largely absent in GATA-3-null follicles.

We were intrigued by the presence of a large group of Lef-1-negative epithelial cells surrounding the Lef-1positive cortical precursor cells near the base of the hair 
bulb (Fig. 6F). Could these Lef-1-negative cells be IRS precursors? To examine this possibility, we took advantage of the functional LacZ coding sequence inserted downstream from the GATA-3 promoter of the KO animals. Previously, we had shown that GATA-3nlslacZ ${ }^{+/-}$ follicles displayed a pattern of $\beta$-galactosidase activity that faithfully paralleled the two rows of anti-GATA-3 immunoreactivity demarcating the Huxley's and cuticle layers of the IRS (see Fig. 2). We now tracked the expression pattern of GATA-3 promoter activity under conditions in which GATA-3 protein was missing. Surprisingly, GATA-3-null follicles displayed a gross expansion of GATA-3-promoter-active ( $\beta$-gal-positive) cells (Fig. 7). Thus, rather than two neat rows as in wild-type follicles (Fig. 7A), extensive GATA-3 promoter activity was detected within cells of the follicle bulbs of GATA-3 protein-deficient follicles (Fig. 7B,C). Most of these cells were negative for AE13 (Fig. 7C) and AE15 staining (see Fig. 6). Thus, neither their position nor their expression pattern was consistent with a precortical identity.

To search for markers that might characterize these GATA-3-promoter-active bulb cells, we examined the expression of FOG1 (riend of GATA- $\underline{1}$ ), which often functions with GATA factors, including GATA-3, to
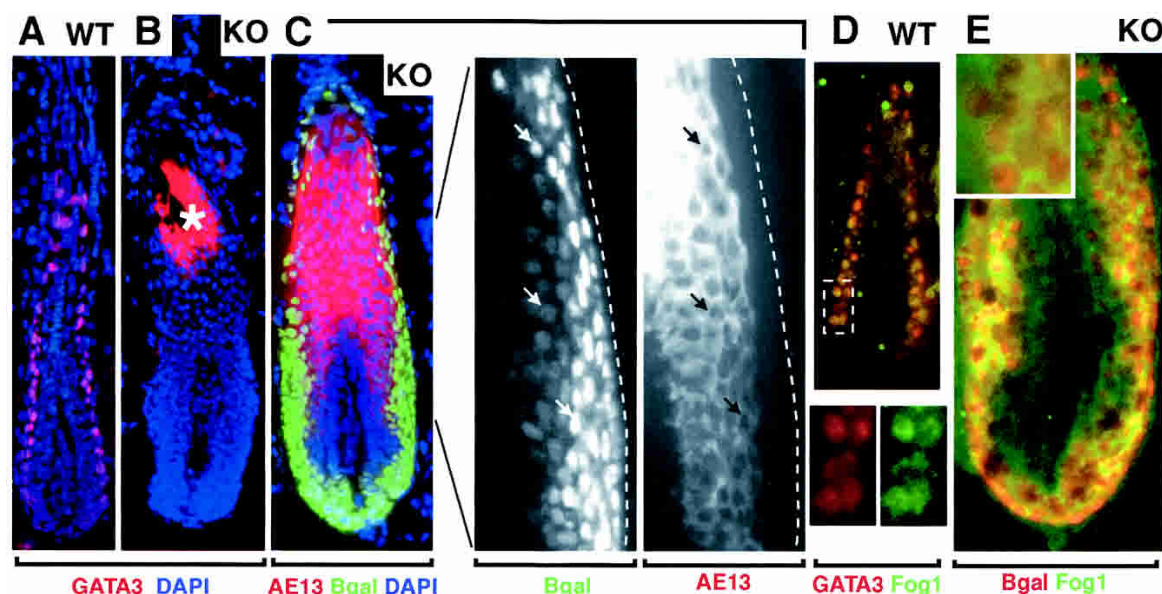

KO F WT G

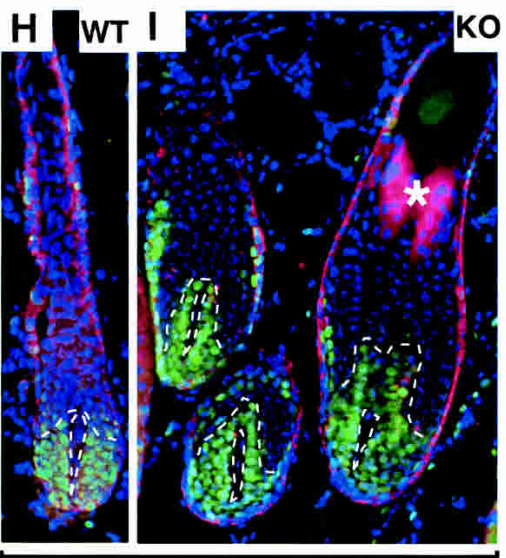

K5 KI67 DAPI

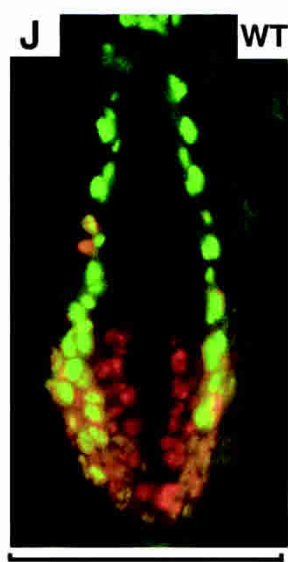

Ki67 GATA3

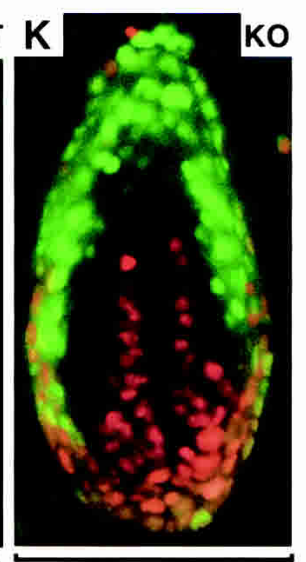

Ki67 Bgal KO
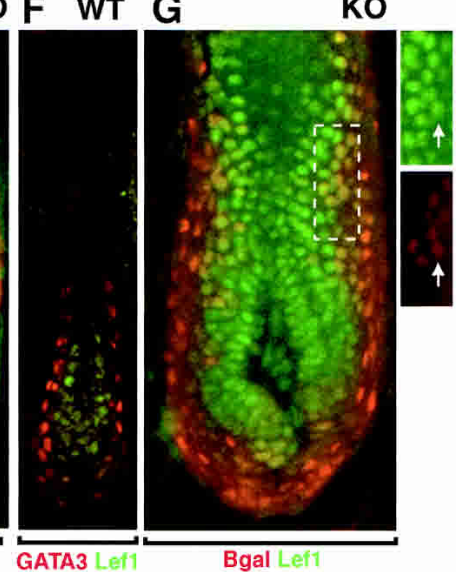

Bgal Lef1

Figure 7. Expansion and aberrancies in expression patterns of cortical and IRS precursor populations in GATA-3-null follicles. Skins from matched P35 wild-type and GATA-3-null grafts were frozen, sectioned (10 $\mu \mathrm{m})$, and subjected to either triple indirect immunofluorescence $(A-K)$ or in situ hybridization $(L, M)$. The genotype of each section is denoted at the upper right. Abs used are color-coded and indicated across the bottom of each frame. Asterisks in $B$ and $I$ denote autofluorescence, an artifact of the knots of highly keratinized material in some KO shafts. Boxed areas are shown at higher magnification in the insets. DAPI (blue) denotes nuclei. Abs were against GATA-3, present in the IRS lineage, and absent in the GATA-3-null sections; $\beta$-galactosidase (Bgal), specific for GATA-3 promoter activity, which was indistinguishable from the anti-GATA IRS labeling in wild-type follicles, and still present in the expanded IRS precursor population of KO follicles; AE13, specific for the keratins of differentiating cortical cells; FOG1, a corepressor for GATA-3 protein, present in IRS precursor cells, where it marked the nuclei of wild-type but the cytoplasm of KO cells ( $D$ and $E$, respectively; see inset to $E$ as well); Lef-1, specific for cortical precursor cells, expanded in the KO; Ki67, specific for proliferating cell nuclei; and K5, specific for the ORS of the follicle. The cRNA probe is against Shh, Sonic hedgehog, which marks a small subset of matrix cells in wild-type follicles, unchanged in KO follicles. Note that in KO follicles, some AE13-positive cortical and Lef-1-positive precortical cells were also positive for GATA-3 promoter activity (representative cells indicated by arrows in $C$ and $G)$. Note also that Ki67 labeling confirms the anagen state of both wild-type and KO follicles, and some Ki67-positive cells were also positive for $G A T A-3$ promoter activity $(J, K)$. 
suppress GATA's action as an activator (Tsang et al. 1997; Zhou et al. 2001). Interestingly, in wild-type follicles, an anti-FOG1 antibody labeled the same two IRS layers that labeled with GATA-3 antibodies (Fig. 7D). Antibodies against GATA-3 and FOG1 both localized to the nuclei of these wild-type IRS precursor cells. In GATA-3-null follicles, anti-FOG1 costained with anti- $\beta$ galactosidase antibodies in the expanded zone of GATA3-promoter-active cells (Fig. 7E). In the absence of GATA-3, however, anti-FOG labeling shifted from nucleus to cytoplasm (Fig. 7E, inset), opening the possibility that GATA-3 and FOG-1 may function together at some point in IRS specification. The increased population of Lef-1-positive precortical cells in GATA-3-null follicles was interesting and suggested an interdependency of the development of IRS and cortical progenitors. Given the marked aberrations in hair shaft, however, we wondered whether the expanded compartments of IRS and cortical precursors were truly normal. Closer inspection of GATA-3-promoter-active cells revealed a number of cells whose nuclei were positive for both $\beta$-galactosidase and cortical keratins (examples marked by arrows in magnified photos in Fig. 7C). Conversely, some of the Lef-1-positive cortical precursor cells were also positive for $\beta$-galactosidase (Fig. 7G, arrows in enlarged photos indicate an example of a cell expressing both Lef- 1 and $\beta$-gal). Such mixing of biochemical markers was not seen in wild-type follicles, where Lef-1-positive precortical cells were distinct in their expression pattern from the compartment of GATA-3/FOG1/GATA-3-promoter-active IRS precursor cells (Figs. 2C, 7F).

Given the expansion of precursor compartments for GATA-3-null IRS and cortex, we examined the status of matrix cells, which are thought to give rise to these precursor populations. In both wild-type and KO follicles, antibodies against the proliferating nuclear antigen, Ki67, revealed intense labeling of the matrix cells, which in anagen, are rapidly proliferating (Fig. 7H,I, green). Overall, the number of Ki67-positive cells was similar in wild-type and $\mathrm{KO}$ follicles.

Finally, we examined the status of Sonic hedgehog (Shh), a regulator of follicle morphogenesis that is normally expressed in a small zone of matrix cells (Gambardella et al. 2000). In mutant mice deficient in the CCATT box displacement factor, CDP, Shh mRNAs are absent from this zone and instead activated in the region corresponding to the IRS (Ellis et al. 2001). Because both CDP and GATA-3 mutations lead to IRS abnormalities, we assessed whether such misregulation might occur in the absence of GATA-3 as well. As shown in Figure 7L and $M$, the expression of Shh mRNAs appeared to be unaffected in the GATA-3 mutant follicles. The significance of this and other differences is discussed in more detail below.

\section{Morphological confirmation of the biochemical defects in GATA-3-null hair follicles}

Ultrastructural analysis of wild-type and GATA-3-null graft skin by transmission electron microscopy helped to further define abnormalities. Figure 8A illustrates representative examples of sections of anagen-phase follicles from age- and litter-matched wild-type and $\mathrm{KO}$ grafted skins. As expected from our analyses of wild-type and GATA-3-null embryonic skins (Fig. 4), differences in epidermises of grafted wild-type and GATA-3-null skin were relatively slight, and expression patterns of cornified envelope proteins and keratins 1 and 5 were similar (data not shown). Morphologically, GATA-3-null epidermis displayed all four stages of terminal differentiation, but the tissue was hyperthickened (see Fig. 8A), consistent with the mild barrier function defect that we had observed earlier (see Fig. 3). More robust signs of barrier defects have been observed in epidermis lacking the Kruppel-like transcription factor, KLF4 (Segre et al. 1999).

Abnormalities were striking between wild-type and KO grafted skins (Fig. 8A). Follicles in wild-type grafted skin developed normally, extending deep into the dermis. In contrast, GATA-3-null follicles and their developing shafts were shorter and fatter, despite expanded compartments of IRS and cortical precursor cells in enlarged bulbs. This appeared to be reflective of defects in both the downward movement of developing follicles as well as upward movement of hair shafts.

At the ultrastructural level, wild-type grafted follicles displayed a normal IRS, with cuticle (Ci), Huxley's (Hu), and Henle's (He) layers readily identified by electrondense trichohyalin granules (Th; Fig. 8B). The Henle's layer is the first IRS layer to accumulate trichohyalin granules, and it eventually keratinizes as it progresses up the follicle (Robins and Breathnach 1970). The opposing arrows in the lower panel of Figure $8 \mathrm{~B}$ denote the juncture between the prekeratinized and keratinized cells of this layer. At the core is the single row of highly organized medulla cells (Me), which also display clusters of trichohyalin granules just below the nucleus (arrowheads in the upper panel of Fig. 8B).

In GATA-3-null follicles, only a single layer of differentiated IRS-like cells was detected (Fig. 8C; double arrows denote the transition to a keratinized Henle's layer-like appearance. Consistent with the anti-trichohyalin labeling (AE15-positive staining, see Fig. 6F), these cells were well above the bulb and contained only a few electron-dense trichohyalin granules (arrowheads in Fig. 8C). Thus, despite a significant pool of IRS precursor cells, morphological as well as biochemical signs of Huxley's layer and IRS cuticle cells were lacking.

Internal to the Henle's layer, only occasional trichohyalin-negative, immature cells were seen in the GATA3-null follicles (Fig. 8C). Instead, most of the central portion of GATA-3-null follicles was packed with keratinized material characteristic of the hair shaft (Fig. 8C). Below the remnants of the Henle's layer were precortical-like cells (PreCo), displaying bundles of keratin filaments (Kfs).

In the shaft area of most GATA-3-null follicles, ORS/ companion layer cells with wisps of keratin filaments abutted against keratin filament-rich precortical-like 
Figure 8. Ultrastructural abnormalities in GATA-3-null follicles. Matched wild-type and GATA-3-null (KO) skin grafts were frozen in OCT $(A, B)$ or fixed, embedded in Epon, and sectioned for ultrathin analyses $(C, D)$. (A) Light microscopic analysis of H\&E-stained 10- $\mu \mathrm{m}$ sections of 5-wk grafts. Wild-type (WT) composite of two $20 \times$ microscopic fields; $\mathrm{KO}$ is visible in one field because of its shorter and fatter structure at all stages of the hair cycle. $\left(B-C^{\prime \prime}\right)$ Ultrastructural analyses of 12-wk grafts. All follicles are oriented with the skin surface at the top. Magnification bars are denoted on each frame. (B) Sagittal section of wild-type follicle, depicting the thin Henle's cell layer (He) with uniform density caused by keratinization. On the outside, this layer is flanked by the companion cell layer $(\mathrm{Cp})$ and ORS and finally the dermal sheath (DS). On the inside, the Henle's layer is flanked by the trichohyalin (Th)-rich Huxley's (Hu) layer of the IRS, and the thin IRS cuticle (Ci). The hair shaft (HS) is internal to the IRS, and is composed of three major layers: an inner core of medulla $(\mathrm{Me})$ cells with trichoyalin granules below each nucleus (arrowheads), the cortex (Co), and the cuticle of the hair shaft (Ch). The upper and lower panels are from the same hair follicle, but the lower panel depicts the base of the keratinized Henle's layer of the IRS (double arrowheads); cells below this juncture still possess trichoyalin granules, but are less mature and less organized, as is characteristic of IRS precursor cells. $\left(C-C^{\prime \prime}\right)$ Sections from GATA-3null follicles. All are views from the region just above the follicle bulb. $(C)$ The origins of the keratinized cells of a Henle's-like layer in a KO follicle (double arrows, IRS ${ }^{\star}$ ). Note the presence of only a few trichoyalin granules (arrowheads) in the IRS precursor cells
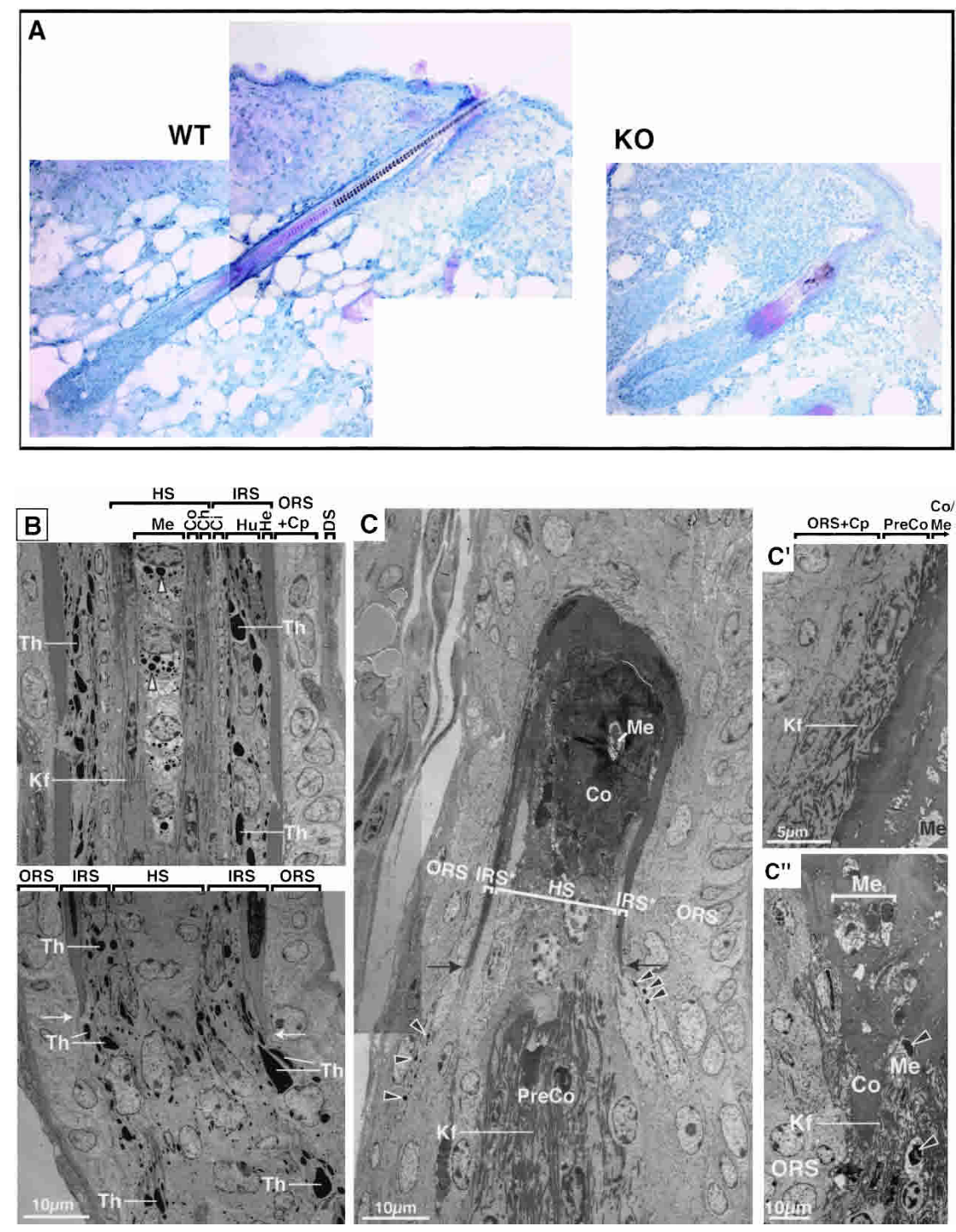

just below these keratinizing cells, in contrast to the wild-type IRS precursors, which are abundant in trichohyalin (Th; lower panel in $B$ ). Note also the presence of precortical cells (PreCo), rich in keratin filaments (Kf), located atypically below the Henle's cells; this irregularity was predicted from our biochemical studies (see Fig. 6F). Note further the mass of highly keratinized cortex and medulla central to the tiny IRS. The sections in $C^{\prime}$ and $C^{\prime \prime}$ show that in some areas, the IRS was missing altogether, and the ORS and companion layer were abutted against the precortex, cortex, and medulla. Again, note signs of gross disorganization, with as many as three medulla cells $\left(\mathrm{Me}\right.$ in $\left.C^{\prime \prime}\right)$ stacked horizontally against each other, instead of in the organized linear vertical array seen in wild-type medulla $(B$, upper panel). Note trichohyalin (arrowheads) in the medulla but not elsewhere $\left(C^{\prime \prime}\right)$.

cells, which, in turn, were adjacent to cortex and medulla (Fig. 8C'). Additionally, medulla cells were markedly disorganized and often embedded in a mass of keratinized cortical cells (Fig. 8C"). Some medulla cells displayed trichohyalin granules (arrowheads in 8C"). However, the organization of granules as well as the organization of cells was aberrant (cf. medulla cells in Fig. $8 \mathrm{C}^{\prime \prime}$ and in $8 \mathrm{~B}$, upper panel). Overall, this analysis confirmed the medulla identity of the thickened core of AE15-positive cells in GATA-3-null follicles (see Fig. 6F) and revealed a level of disorganization of the hair shaft that was even more prominent than that envisioned from the immunohistochemical studies.

\section{Discussion}

With each new hair cycle, the IRS and hair shaft must develop simultaneously to provide a channel for the new hair to emerge. Yet despite significant advances in our understanding of the molecular controls governing hair shaft differentiation, little is known about the regulation and function of the IRS. Our present studies have now uncovered a member of the well-studied GATA family of transcription factors as a major and essential regulator of IRS differentiation.

The structural consequences of IRS loss are severe. Without the Huxley's and cuticle layers, the Henle's 
layer does not develop properly, and without the IRS, gross abnormalities arise in composition and organization of cells within the hair shaft. Thus, not only are the cortex and medulla dramatically thickened, but in addition, cells no longer spatially align. The shaft develops as a hyperthickened mass of irregular medulla, cortical, and cuticle cells, rather than the architectural wonder of a normal shaft. Perhaps as no other knockout has so graphically illustrated, these two thin layers of IRS cells are critical for the proper differentiation and/or organization of the hair shaft.

In recent years, a myriad of genes encoding transcription factors have been genetically implicated in follicle development (Cachon-Gonzalez et al. 1994; Nehls et al. 1994; van Genderen et al. 1994; Segre et al. 1995; Zhou et al. 1995; Dai et al. 1998; Li et al. 2000; Pennisi et al. 2000; Ellis et al. 2001; Ma et al. 2003; Mill et al. 2003). When mutated, only a few of these genes result in defects involving the IRS. Of these, hairless (encoding a zinc finger protein required for postnatal hair cycling) and nude (encoding foxn1, a forkhead protein) are expressed in both hair shaft and IRS precursors, and display defects in both (Cachon-Gonzalez et al. 1994; Nehls et al. 1994; Segre et al. 1995; Lee et al. 1999; Panteleyev et al. 2000). In contrast, although GATA-3's absence resulted in major abnormalities in hair structure, involving both IRS and shaft, it was only expressed in two of the IRS layers. This enabled us to conclude that the severe hair shaft defects that occurred in the GATA-3-deficient mouse originated specifically from transcriptional aberrations within the IRS.

To date, the highly restricted pattern of GATA-3 expression is unique, but it is most similar to that of the transcriptional repressor $\mathrm{CDP}$, which represses genes regulated by CCAAT sequence motifs (Ellis et al. 2001). The Cutl1 gene encoding CDP seemed to be expressed earlier and more broadly in the IRS pathway, where it was found in the outermost layer of the lower ORS, in IRS progenitor cells of the matrix, and in all three IRS layers. A few mice harboring mutations in Cutl1 survived their lung defects long enough to exhibit a sparse and abnormal hair coat. The morphology of Cut11-null hair follicles was unusual, as just above a relatively normally structured bulb, the IRS formed, but then both IRS and shaft eventually degenerated to form a large follicular cyst, soon appearing more similar to that of nude or even hairless mutant mice (Ellis et al. 2001).

Although both Cutl1- and GATA-3-null follicles exhibited a failure to develop a proper IRS, GATA-3's absence was felt most strongly in two major ways that involve cell fate specification within the hair follicle. First, it resulted in an accumulation of IRS precursor cells that appeared to be dysfunctional, unable to differentiate into trichohyalin- and keratin-producing IRS cells. Second, it led to an expansion of seemingly functional Lef-1-expressing precortical cells, which differentiated into hair keratin-producing cortical cells. Some of these Lef-1-positive cells exhibited mixing of IRS and cortical gene expression patterns (Fig. 6C,E). Because we did not detect such cells in wild-type, GATA-3-contain- ing follicles (Figs. 2C, 6F), these findings support the argument that the loss of GATA-3 results not only in expansion of selective cell populations within the follicle, but also in some misexpression of genes in these compartments. In contrast, Cut1-null follicles exhibited substantial misexpression of both trichohyalin and the companion layer $\mathrm{K} 6$ in the cortex, features that were not observed in GATA-3-null follicles, as well as strong expression of the Cutl1 promoter throughout the cortex. Furthermore, in Cut1-null vibrissae, Shh was aberrantly expressed in the IRS (Ellis et al. 2001), whereas in GATA-3 mutant mice, Shh expression appeared unaffected (Fig. 7L,M). It may still be possible that CDP and GATA-3 function in the same pathway to regulate IRS specification, but the target genes they affect must be at least partially nonoverlapping.

It is interesting that in T-cell development, both GATA-3 and Lef-1/Tcf1 are necessary early to direct lymphoid precursors to become T cells, and later in the lineage, GATA-3 acts independently of Lef- 1 in Th cell commitment (van Genderen et al. 1994; Verbeek et al. 1995; Hattori et al. 1996; Ting et al. 1996; Okamura et al. 1998; Schilham et al. 1998; Hendriks et al. 1999). In naive Th cells, FOG1 and GATA-3 act to inhibit interleukin gene expression, and upon Th commitment, FOG1 is down-regulated (Kurata et al. 2002). GATA-3, which autoactivates its own expression, is required for Th2 cell differentiation (Ouyang et al. 2000; Zhou et al. 2001), whereas suppression of GATA-3 promotes Th1 cell determination (for review, see Patient and McGhee 2002).

Despite some differences, the transcriptional parallels between $\mathrm{T}$-cell development and hair follicle differentiation are quite striking. In the postnatal hair follicle, Lef$1 / \beta$-catenin and GATA-3 seem to act in parallel but distinct pathways governing cortical and IRS differentiation, respectively. The expansion of the cortical lineage in the GATA-3-deficient state could be analogous to an increase in Th1 rather than Th2 cell differentiation, and the up-regulation of GATA-3 promoter activity in GATA-3-deficient IRS precursor cells might be a sign that FOG1 and GATA-3 normally repress this promoter in wild-type IRS precursors. When taken together with the paucity of differentiated IRS cells, the expanded population of GATA-3-promoter-active/FOG1-positive cells in GATA-3-deficient follicles might represent a population of stalled IRS precursors. The enhanced GATA-3 immunoreactivity as IRS precursor cells differentiate into IRS cells in wild-type skin is consistent with a transactivating role for GATA-3 later in IRS differentiation, perhaps analogous to Th2 cell differentiation. The fact that the structural genes for IRS differentiation are not expressed in the GATA-3-deficient state, despite the presence of progenitor cells, further supports a positive role for GATA-3 in their expression.

In summary, our studies illuminate a new dimension in our understanding of how hair follicle morphogenesis is transcriptionally controlled. In many respects, GATA-3 appears to be a master regulator of IRS cell lineage determination as Lef- $1 / \beta$-catenin is for cortical lineage specification. The matrix offers a rich milieu of en- 
vironmental cues that ultimately lead to the differential specification of these lineages. In the future, our efforts will be placed on understanding how these signals impact on GATA-3 expression as well as identifying the downstream target genes for this novel and important regulator of IRS differentiation.

\section{Materials and methods}

Mice

The generation and characterization of the GATA-3nlslacZ mice has been described (Hendriks et al. 1999; van Doorninck et al. 1999). Briefly, the lacZ gene fused to a nuclear localization signal (nls) was placed in-frame at the ATG translational start site in the GATA-3 locus, inactivating the GATA-3 gene and expressing $\beta$-galactosidase from the endogenous GATA-3 promoter. To obtain GATA-3-null embryos that survive to E18.5, we used a modification of a drug rescue regime (Lim et al. 2000; K.C. Lim, pers. comm.). Beginning at E7.5, pregnant GATA3nlslacZ/+ dams that were mated to GATA-3nlslacZ/+ males were given fresh water daily containing $100 \mu \mathrm{g} / \mathrm{mL}$ L-phenylephrine (Sigma P6126), $100 \mu \mathrm{g} / \mathrm{mL}$ isoproterenol (Sigma 15627) and $2 \mathrm{mg} / \mathrm{mL}$ ascorbic acid (Sigma A0278). The presence of the GATA-3nlslacZ allele was determined by a combination of PCR screening, X-gal staining, phenotypic identification of GATA-3null embryos, and/or loss of GATA-3 immunoreactivity (Lim et al. 2000). The PCR primers used were $5^{\prime}$-TCCTGCGAGCCTG GCTGTCGGA-3', which recognizes GATA-3 intron $1 ; 5^{\prime}$-CCT GTAGCCAGCTTTCATAAC-3', which recognizes $1 a c Z$; and 5'-GTTGCCTTGACCATCGATGTT-3', which recognizes GATA-3 exon 2. The reaction conditions were $94^{\circ} \mathrm{C}$ for $5 \mathrm{~min}$, followed by 45 cycles of $94^{\circ} \mathrm{C}$ for $30 \mathrm{sec}, 53^{\circ} \mathrm{C}$ for $30 \mathrm{sec}$, and $72^{\circ} \mathrm{C}$ for $1.2 \mathrm{~min}$. The band sizes are: $\sim 750 \mathrm{bp}$ (wild-type allele) and $\sim 1.1 \mathrm{~kb}$ (GATA-3nlslacz allele).

\section{Histology and electron microscopy}

Tissues for immunofluorescence and $H \& E$ staining were embedded in OCT and then frozen immediately on dry ice. Tissues for scanning electron microscopy (EM) were fixed in $2.5 \%$ glutaraldehyde in PBS at room temperature for $>1 \mathrm{~h}$, processed using standard techniques (Fujiwara et al. 2002), and visualized using a JEOL JSM-35 scanning electron microscope. For transmission EM, samples were fixed in $2 \%$ glutaraldehyde, $4 \%$ formaldehyde, and $2 \mathrm{mM} \mathrm{CaCl}_{2}$ in $0.05 \mathrm{M}$ sodium cacodylate buffer at room temperature for $>1 \mathrm{~h}$ and processed for Epon embedding as described (Segre et al. 1999). Samples were visualized with a Tecnai G2 transmission electron microscope.

\section{In situ analysis}

Digoxygenin probe synthesis was performed according to the manufacturer's instructions (Roche). Whole mount in situ hybridization was described by Conlon and Rossant (1992), with modifications (Byrne et al. 1994). In situ hybridizations on 10$\mu \mathrm{m}$ (Shh) and 15- $\mu \mathrm{m}$ (GATA-3) frozen sections were performed as described (Schaeren-Wiemers and Gerfin-Moser 1993).

\section{$X$-gal staining and immunofluorescence}

OCT sections were fixed for $30 \mathrm{sec}$ in $0.1 \%$ glutaraldehyde, washed three times in PBS, and incubated in X-gal staining solution at $37^{\circ} \mathrm{C}$ for $1-2 \mathrm{~h}$. OCT sections were then fixed for 10 $\mathrm{min}$ in $4 \%$ PFA in PBS and washed four times for $5 \mathrm{~min}$ in PBS.
When staining with mouse monoclonal antibodies, we used the reagents and protocol from the MOM Basic kit (Vector Labs). In other cases, the following block/diluent was used: $2.5 \%$ NDS, $2.5 \%$ NGS, $1 \%$ BSA, $2 \%$ gelatin, and $1 \%$ Triton X-100 in PBS. The primary antibodies at the indicated concentrations were AE13 (mouse, 1:50-1:100; Lynch et al. 1986), AE15 (mouse, 1: 10; O'Guin et al. 1992), K5 (gp, 1:250; Fuchs lab), GATA-3 (mouse, 1:100; Santa Cruz, HCG3-31), $\beta$-galactosidase (rabbit, 1:400; mouse, 1:100; Harlan; Sigma), K1 (rabbit, 1:250; Fuchs lab), loricrin (rabbit, 1:300; Fuchs lab), Lef-1 (rabbit, 1:250; Fuchs lab), filaggrin (rabbit, 1:1000; Covance, PRB-417P), K6 (rabbit, 1:500; Fuchs lab), involucrin (mouse, 1:200; Babco), Ki67 (rabbit, 1:1000; NovoCastra Laboratories Ltd.), and FOG1 (goat, 1:50; Santa Cruz, sc-9361). Relevant FITC- or TxR-conjugated donkey or goat antibodies (1:100; Jackson Laboratories) were used for detection of primary antibodies.

\section{Barrier function assay}

Embryos were submerged at $37^{\circ} \mathrm{C}$ for $>8 \mathrm{~h}$ in a solution of 1.3 $\mathrm{mM} \mathrm{MgCl} 2,100 \mathrm{mM} \mathrm{NaPO}_{4}, 3 \mathrm{mM} \mathrm{K} \mathrm{K}_{3} \mathrm{Fe}(\mathrm{CN})_{6}, 3 \mathrm{mM}$ $\mathrm{K}_{4} \mathrm{Fe}(\mathrm{CN})_{6}, 0.01 \%$ sodium deoxycholate, $0.2 \% \mathrm{NP}-40$, and 1 $\mathrm{mg} / \mathrm{mL}$ X-gal, which was adjusted to a $\mathrm{pH}$ of 4.5 with $\mathrm{HCl}$ (Hardman et al. 1998). At this $\mathrm{pH}$ in the absence of the epidermal barrier, the solution penetrates epidermis, and an endogenous $\beta$-galactosidase-like activity catalyzes production of a blue precipitate.

\section{Skin grafting}

Pregnant GATA-3nlslacZ/+ dams were killed at E17.5, and embryos were removed. Full thickness skins were removed from torsos of wild-type and GATA-3-null embryos, spread on a sterile plastic dish, and stored briefly at $4^{\circ} \mathrm{C}$. During this time, each skin graft recipient site was prepared by removal of a patch of full thickness skin on an anesthetized female $n u / n u C D-1$ mouse. Embryonic donor skin was then placed on the graft bed and secured by sterile gauze and cloth bandages. A total of 12 grafts were placed (six wild-type and six GATA-3-null), and each showed a consistent phenotype dependent on the presence or absence of GATA-3 in the donor skin.

\section{Acknowledgments}

A portion of this research was conducted at The University of Chicago, where C.K. and D.B. are enrolled as $\mathrm{MD} / \mathrm{Ph} . \mathrm{D}$ students. We thank Frank Grosveld (Erasmus University, Rotterdam, The Netherlands) for GATA-3nlslac $Z^{+/-}$mice, Linda Degenstein and Lisa Polak for assistance with mouse husbandry, T.T. Sun (NY University School of Medicine) for AE13 and AE15 antibodies, R. Cannon (University of North Carolina, Chapel Hill, NC) for the GATA-3 probe, C. Tabin (Harvard Medical School, Boston, MA) for the Shh probe, and N. Lampen (EM facility at Memorial Sloan-Kettering Cancer Center) for assistance with scanning electron microscopy. C.K. and D.B. are supported by the Medical Scientist National Research Service Award, NIH/National Institute of General Medical Sciences, Grant No. 5 T32 GM07281. D.B. also receives support from The University of Chicago, Division of Biological Sciences, Naomi Ragins Goldsmith Fund. K.-C.L. is supported by NIH Grant GM 28896. E.F. is an HHMI Investigator. This research was funded by a grant from the National Institutes of Health.

The publication costs of this article were defrayed in part by payment of page charges. This article must therefore be hereby 
marked "advertisement" in accordance with 18 USC section 1734 solely to indicate this fact.

\section{References}

Byrne, C., Tainsky, M., and Fuchs, E. 1994. Programming gene expression in developing epidermis. Development 120: $2369-2383$.

Cachon-Gonzalez, M.B., Fenner, S., Coffin, J.M., Moran, C., Best, S., and Stoye, J.P. 1994. Structure and expression of the hairless gene of mice. Proc. Natl. Acad. Sci. 91: 7717-7721.

Cantor, A.B. and Orkin, S.H. 2002. Transcriptional regulation of erythropoiesis: An affair involving multiple partners. Oncogene 21: 3368-3376.

Carlsson, P., Waterman, M.L., and Jones, K.A. 1993. The hLEF/ TCF-1 $\alpha$ HMG protein contains a context-dependent transcriptional activation domain that induces the TCR $\alpha$ enhancer in T cells. Genes \& Dev. 7: 2418-2430.

Chan, E.F., Gat, U., McNiff, J.M., and Fuchs, E. 1999. A common human skin tumour is caused by activating mutations in $\beta$-catenin. Nat. Genet. 21: 410-413.

Conlon, R.A. and Rossant, J. 1992. Exogenous retinoic acid rapidly induces anterior ectopic expression of murine Hox-2 genes in vivo. Development 116: 357-368.

Cotsarelis, G., Sun, T.T., and Lavker, R.M. 1990. Label-retaining cells reside in the bulge area of pilosebaceous unit: Implications for follicular stem cells, hair cycle, and skin carcinogenesis. Cell 61: 1329-1337.

Dai, X., Schonbaum, C., Degenstein, L., Bai, W., Mahowald, A., and Fuchs, E. 1998. The ovo gene required for cuticle formation and oogenesis in flies is involved in hair formation and spermatogenesis in mice. Genes \& Dev. 12: 3452-3463.

DasGupta, R. and Fuchs, E. 1999. Multiple roles for activated LEF/TCF transcription complexes during hair follicle development and differentiation. Development 126: 4557-4568.

Dry, F.W. 1926. The coat of the mouse (Mus musculum). I. Genet. 16: 287-340.

Ellis, T., Gambardella, L., Horcher, M., Tschanz, S., Capol, J., Bertram, P., Jochum, W., Barrandon, Y., and Busslinger, M. 2001. The transcriptional repressor CDP (Cutl1) is essential for epithelial cell differentiation of the lung and the hair follicle. Genes \& Dev. 15: 2307-2319.

Flanagan, S.P. 1966. 'Nude,' a new hairless gene with pleiotropic effects in the mouse. Genet. Res. 8: 295-309.

Fuchs, E., Merrill, B.J., Jamora, C., and DasGupta, R. 2001. At the roots of a never-ending cycle. Dev. Cell 1: 13-25.

Fujiwara, T., Dehart, D.B., Sulik, K.K., and Hogan, B.L. 2002. Distinct requirements for extra-embryonic and embryonic bone morphogenetic protein 4 in the formation of the node and primitive streak and coordination of left-right asymmetry in the mouse. Development 129: 4685-4696.

Gambardella, L., Schneider-Maunoury, S., Voiculescu, O., Charnay, P., and Barrandon, Y. 2000. Pattern of expression of the transcription factor Krox-20 in mouse hair follicle. Mech. Dev. 96: 215-218.

Gat, U., DasGupta, R., Degenstein, L., and Fuchs, E. 1998. De novo hair follicle morphogenesis and hair tumors in mice expressing a truncated $\beta$-catenin in skin. Cell 95: 605-614.

Giese, K. and Grosschedl, R. 1993. LEF-1 contains an activation domain that stimulates transcription only in a specific context of factor-binding sites. EMBO J. 12: 4667-4676.

Hardman, M.J., Sisi, P., Banbury, D.N., and Byrne, C. 1998. Patterned acquisition of skin barrier function during development. Development 125: 1541-1552.

Hardy, M.H. 1992. The secret life of the hair follicle. Trends
Genet. 8: 55-61.

Hattori, N., Kawamoto, H., Fujimoto, S., Kuno, K., and Katsura, Y. 1996. Involvement of transcription factors TCF-1 and GATA-3 in the initiation of the earliest step of T cell development in the thymus. J. Exp. Med. 184: 1137-1147.

Hendriks, R.W., Nawijn, M.C., Engel, J.D., van Doorninck, H., Grosveld, F., and Karis, A. 1999. Expression of the transcription factor GATA-3 is required for the development of the earliest $\mathrm{T}$ cell progenitors and correlates with stages of cellular proliferation in the thymus. Eur. J. Immunol. 29: 19121918.

Kuo, C.T. and Leiden, J.M. 1999. Transcriptional regulation of T lymphocyte development and function. Annu. Rev. Immunol. 17: 149-187.

Kurata, H., Lee, H.J., McClanahan, T., Coffman, R.L., O'Garra, A., and Arai, N. 2002. Friend of GATA is expressed in naive Th cells and functions as a repressor of GATA-3-mediated Th2 cell development. J. Immunol. 168: 4538-4545.

Lakshmanan, G., Lieuw, K.H., Lim, K.C., Gu, Y., Grosveld, F., Engel, J.D., and Karis, A. 1999. Localization of distant urogenital system-, central nervous system-, and endocardiumspecific transcriptional regulatory elements in the GATA-3 locus. Mol. Cell. Biol. 19: 1558-1568.

Langbein, L., Rogers, M.A., Praetzel, S., Winter, H., and Schweizer, J. 2003. K6irs1, K6irs2, K6irs3, and K6irs4 represent the inner-root-sheath-specific type II epithelial keratins of the human hair follicle. J. Invest. Dermatol. 120: 512-522.

Lee, D., Prowse, D.M., and Brissette, J.L. 1999. Association between mouse nude gene expression and the initiation of epithelial terminal differentiation. Dev. Biol. 208: 362-374.

Li, M., Indra, A.K., Warot, X., Brocard, J., Messaddeq, N., Kato, S., Metzger, D., and Chambon, P. 2000. Skin abnormalities generated by temporally controlled $\operatorname{RXR} \alpha$ mutations in mouse epidermis. Nature 407: 633-636.

Lim, K.C., Lakshmanan, G., Crawford, S.E., Gu, Y., Grosveld, F., and Engel, J.D. 2000. Gata3 loss leads to embryonic lethality due to noradrenaline deficiency of the sympathetic nervous system. Nat. Genet. 25: 209-212.

Lynch, M.H., O'Guin, W.M., Hardy, C., Mak, L., and Sun, T.T. 1986. Acidic and basic hair/nail ("hard") keratins: Their colocalization in upper cortical and cuticle cells of the human hair follicle and their relationship to "soft" keratins. I. Cell Biol. 103: 2593-2606.

Ma, L., Liu, J., Wu, T., Plikus, M., Jiang, T.X., Bi, Q., Liu, Y.H., Muller-Rover, S., Peters, H., Sundberg, J.P., et al. 2003. 'Cyclic alopecia' in Msx2 mutants: Defects in hair cycling and hair shaft differentiation. Development 130: 379-389.

Mill, P., Mo, R., Fu, H., Grachtchouk, M., Kim, P.C., Dlugosz, A.A., and Hui, C.C. 2003. Sonic hedgehog-dependent activation of Gli2 is essential for embryonic hair follicle development. Genes \& Dev. 17: 282-294.

Millar, S.E. 2002. Molecular mechanisms regulating hair follicle development. J. Invest. Dermatol. 118: 216-225.

Nehls, M., Pfeifer, D., Schorpp, M., Hedrich, H., and Boehm, T. 1994. New member of the winged-helix protein family disrupted in mouse and rat nude mutations. Nature 372: 103 107.

Niemann, C. and Watt, F.M. 2002. Designer skin: Lineage commitment in postnatal epidermis. Trends Cell Biol. 12: 185192.

O'Guin, W.M., Sun, T.T., and Manabe, M. 1992. Interaction of trichohyalin with intermediate filaments: Three immunologically defined stages of trichohyalin maturation. J. Invest. Dermatol. 98: 24-32.

Okamura, R.M., Sigvardsson, M., Galceran, J., Verbeek, S., Clevers, H., and Grosschedl, R. 1998. Redundant regulation 
Kaufman et al.

of $\mathrm{T}$ cell differentiation and TCR $\alpha$ gene expression by the transcription factors LEF-1 and TCF-1. Immunity 8: 11-20.

Oliver, R.F. and Jahoda, C.A. 1988. Dermal-epidermal interactions. Clin. Dermatol. 6: $74-82$.

Oosterwegel, M., Timmerman, J., Leiden, J., and Clevers, H. 1992. Expression of GATA-3 during lymphocyte differentiation and mouse embryogenesis. Dev. Immunol. 3: 1-11.

Oshima, H., Rochat, A., Kedzia, C., Kobayashi, K., and Barrandon, Y. 2001. Morphogenesis and renewal of hair follicles from adult multipotent stem cells. Cell 104: 233-245.

Ouyang, W., Lohning, M., Gao, Z., Assenmacher, M., Ranganath, S., Radbruch, A., and Murphy, K.M. 2000. Stat6-independent GATA-3 autoactivation directs IL-4-independent Th2 development and commitment. Immunity 12: 27-37.

Pandolfi, P.P., Roth, M.E., Karis, A., Leonard, M.W., Dzierzak, E., Grosveld, F.G., Engel, J.D., and Lindenbaum, M.H. 1995. Targeted disruption of the GATA3 gene causes severe abnormalities in the nervous system and in fetal liver haematopoiesis. Nat. Genet. 11: 40-44.

Panteleyev, A.A., Paus, R., and Christiano, A.M. 2000. Patterns of hairless (hr) gene expression in mouse hair follicle morphogenesis and cycling. Am. J. Pathol. 157: 1071-1079.

Panteleyev, A.A., Jahoda, C.A., and Christiano, A.M. 2001. Hair follicle predetermination. J. Cell Sci. 114: 3419-3431.

Patient, R.K. and McGhee, J.D. 2002. The GATA family (vertebrates and invertebrates). Curr. Opin. Genet. Dev. 12: 416422.

Pennisi, D., Bowles, J., Nagy, A., Muscat, G., and Koopman, P. 2000. Mice-null for sox18 are viable and display a mild coat defect. Mol. Cell. Biol. 20: 9331-9336.

Robins, E.J. and Breathnach, A.S. 1970. Fine structure of bulbar end of human foetal hair follicle at stage of differentiation of inner root sheath. J. Anat. 107: 131-146.

Schaeren-Wiemers, N. and Gerfin-Moser, A. 1993. A single protocol to detect transcripts of various types and expression levels in neural tissue and cultured cells: In situ hybridization using digoxigenin-labelled cRNA probes. Histochemistry 100: 431-440.

Schilham, M.W., Wilson, A., Moerer, P., Benaissa-Trouw, B.J., Cumano, A., and Clevers, H.C. 1998. Critical involvement of Tcf-1 in expansion of thymocytes. J. Immunol. 161: 39843991.

Segre, J.A., Nemhauser, J.L., Taylor, B.A., Nadeau, J.H., and Lander, E.S. 1995. Positional cloning of the nude locus: Genetic, physical, and transcription maps of the region and mutations in the mouse and rat. Genomics 28: 549-559.

Segre, J.A., Bauer, C., and Fuchs, E. 1999. Klf4 is a transcription factor required for establishing the barrier function of the skin. Nat. Genet. 22: 356-360.

Taylor, G., Lehrer, M.S., Jensen, P.J., Sun, T.T., and Lavker, R.M. 2000. Involvement of follicular stem cells in forming not only the follicle but also the epidermis. Cell 102: 451461.

Ting, C.N., Olson, M.C., Barton, K.P., and Leiden, J.M. 1996. Transcription factor GATA-3 is required for development of the T-cell lineage. Nature 384: 474-478.

Tsang, A.P., Visvader, J.E., Turner, C.A., Fujiwara, Y., Yu, C., Weiss, M.J., Crossley, M., and Orkin, S.H. 1997. FOG, a multitype zinc finger protein, acts as a cofactor for transcription factor GATA-1 in erythroid and megakaryocytic differentiation. Cell 90: 109-119.

van Doorninck, J.H., van Der Wees, J., Karis, A., Goedknegt, E., Engel, J.D., Coesmans, M., Rutteman, M., Grosveld, F., and De Zeeuw, C.I. 1999. GATA-3 is involved in the development of serotonergic neurons in the caudal raphe nuclei. $J$. Neurosci. 19: RC12. van Genderen, C., Okamura, R.M., Farinas, I., Quo, R.G., Parslow, T.G., Bruhn, L., and Grosschedl, R. 1994. Development of several organs that require inductive epithelial-mesenchymal interactions is impaired in LEF-1-deficient mice. Genes \& Dev. 8: 2691-2703.

Verbeek, S., Izon, D., Hofhuis, F., Robanus-Maandag, E., te Riele, H., van de Wetering, M., Oosterwegel, M., Wilson, A., MacDonald, H.R., and Clevers, H. 1995. An HMG-box-containing T-cell factor required for thymocyte differentiation. Nature 374: 70-74.

Winter, H., Langbein, L., Praetzel, S., Jacobs, M., Rogers, M.A., Leigh, I.M., Tidman, N., and Schweizer, J. 1998. A novel human type II cytokeratin, K6hf, specifically expressed in the companion layer of the hair follicle. J. Invest. Dermatol. 111: 955-962.

Zhang, D.H., Cohn, L., Ray, P., Bottomly, K., and Ray, A. 1997. Transcription factor GATA-3 is differentially expressed in murine Th1 and Th2 cells and controls Th2-specific expression of the interleukin-5 gene. J. Biol. Chem. 272: 2159721603.

Zheng, W. and Flavell, R.A. 1997. The transcription factor GATA-3 is necessary and sufficient for Th2 cytokine gene expression in CD4 T cells. Cell 89: 587-596.

Zhou, M., Ouyang, W., Gong, Q., Katz, S.G., White, J.M., Orkin, S.H., and Murphy, K.M. 2001. Friend of GATA-1 represses GATA-3-dependent activity in CD4+ T cells. J. Exp. Med. 194: $1461-1471$.

Zhou, P., Byrne, C., Jacobs, J., and Fuchs, E. 1995. Lymphoid enhancer factor 1 directs hair follicle patterning and epithelial cell fate. Genes \& Dev. 9: 700-713. 


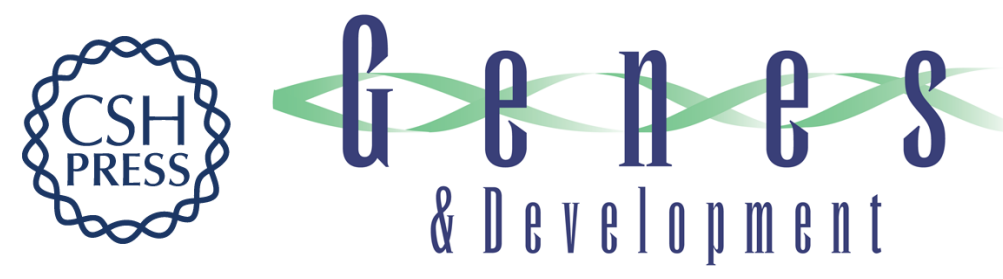

\section{GATA-3: an unexpected regulator of cell lineage determination in skin}

Charles K. Kaufman, Ping Zhou, H. Amalia Pasolli, et al.

Genes Dev. 2003, 17:

Access the most recent version at doi:10.1101/gad.1115203

References This article cites 59 articles, 21 of which can be accessed free at: http://genesdev.cshlp.org/content/17/17/2108.full.html\#ref-list-1

License

Email Alerting Receive free email alerts when new articles cite this article - sign up in the box at the top Service right corner of the article or click here.

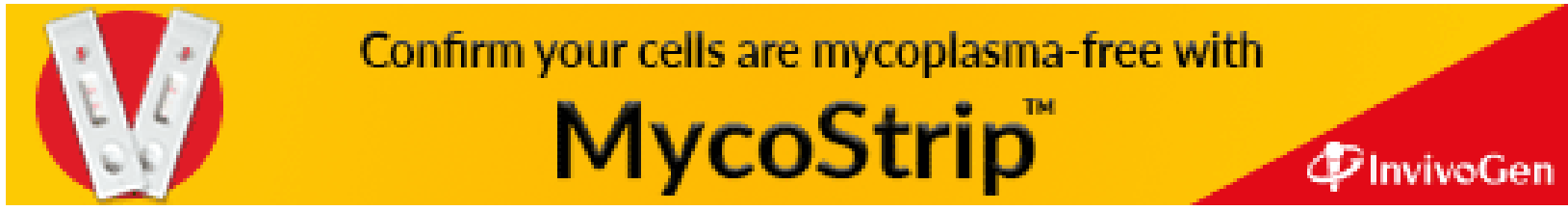

